\title{
Observables of low-lying supersymmetric vectorlike leptonic generations via loop corrections
}

\author{
Amin Aboubrahim, ${ }^{1, *}$ Tarek Ibrahim, ${ }^{2, \dagger, *}$ Ahmad Itani, ${ }^{3, \S}$ and Pran Nath ${ }^{1, \|}$ \\ ${ }^{1}$ Department of Physics, Northeastern University, Boston, Massachusetts 02115-5000, USA \\ ${ }^{2}$ University of Science and Technology, Zewail City of Science and Technology, \\ 6th of October City, Giza 12588, Egypt \\ ${ }^{3}$ Department of Physics, Faculty of Science, The International University of Beirut, \\ Beirut 146404 Mazraa, Lebanon
}

(Received 1 August 2018; published 12 October 2018)

\begin{abstract}
A correlated analysis of observables arising from loop induced effects from a vectorlike generation is given. The observables include flavor changing radiative decays $\mu \rightarrow e \gamma, \tau \rightarrow \mu \gamma, \tau \rightarrow e \gamma$, electric dipole moments of the charged leptons $e, \mu, \tau$, and corrections to magnetic dipole moments of $g_{\mu}-2$ and $g_{e}-2$. In this work we give a full analysis of the corrections to these observables by taking into account both the supersymmetry loops as well as the exchange of a vectorlike leptonic generation. Thus the fermion mass matrix involves a $5 \times 5$ mixing matrix while the scalar sector involves a $10 \times 10$ mixing matrix including the $C P$ violating phases from the vectorlike sector. The analysis is done under the constraint of the Higgs boson mass at the experimentally measured value. The loops considered include the exchange of $W$ and $Z$ bosons and of leptons and a mirror lepton, and the exchange of charginos and neutralinos, sleptons and mirror sleptons. The correction to the diphoton decay of the Higgs $h \rightarrow \gamma \gamma$ including the exchange of the vectorlike leptonic multiplet is also computed.
\end{abstract}

DOI: 10.1103/PhysRevD.98.075009

\section{INTRODUCTION}

Precision measurements can reveal small deviations from the standard model (SM) prediction and indicate the existence of new physics beyond the standard model. There are a variety of experiments which are exploring the properties of elementary particles to a high precision to this end. These include flavor changing radiative decays of the charged leptons $\mu \rightarrow e \gamma, \tau \rightarrow \mu \gamma$ and $\tau \rightarrow e \gamma$, i.e., the MEG experiment [1], BABAR collaboration [2] and the Belle collaboration [3], the electric dipole moment (EDM) of the electron [4], of the muon as well as of quarks [5], and the precision measurement of the anomalous magnetic moment of the muon [6] and of the electron. In this work we explore the implications of a low-lying vectorlike generation on the leptonic processes mentioned above. Vectorlike generations exist in a variety of models including grand

\footnotetext{
*a.abouibrahim@northeastern.edu

†Permanent address: Department of Physics, Faculty of Science, University of Alexandria, Alexandria, Egypt.

*tibrahim@ zewailcity.edu.eg

\$ahmad.itani@liu.edu.lb

"nath@neu.edu

Published by the American Physical Society under the terms of the Creative Commons Attribution 4.0 International license. Further distribution of this work must maintain attribution to the author(s) and the published article's title, journal citation, and DOI. Funded by SCOAP.
}

unified models, string models and D brane models [7-9]. Some of these vectorlike generations may be light. Further, vectorlike generations are anomaly free so they preserve good properties of the model as a quantum field theory. The mixings of these light vectorlike generations with the three generations of leptons can lead to contributions to the processes noted above. Several studies of the effects of vectorlike leptons in various processes already exist [10-20] and in nonsupersymmetric context in [21-27]. In this analysis we perform a correlated study of the contributions of the vectorlike generation to these phenomena. The analysis involves an enlarged leptonic mass matrix which is $5 \times 5$ and a slepton mass-squared matrix which is $10 \times 10$ including the $C P$ violating phases from the vectorlike sector. In the analysis we consider loop exchange of $W$ and $Z$ bosons, leptons and mirror leptons, and exchange of charginos and neutralinos along with the sleptons and mirror sleptons. The analysis is done under the constraint of the Higgs boson mass at $\sim 125 \mathrm{GeV}$, and an analysis of the contribution to the branching ratio $h \rightarrow \gamma \gamma$ from the vectorlike leptonic exchange is also given.

The outline of the rest of the paper is as follows: In Sec. II we give a description of the model. In Sec. III we give an analysis of the flavor changing decays of the charged leptons. An analysis of the EDM of the charged leptons is given in Sec. IV. In Sec. V we give an analysis of $g-2$ for the charged leptons. An analysis of the contribution of the vectorlike leptonic generation to the diphoton decay of the Higgs boson 
is given in Sec. VI. A numerical analysis is given in Sec. VII and conclusions are given in Sec. VIII. Further details of the analysis is given in Appendices A and B.

\section{DESCRIPTION OF THE MODEL}

In this section we give details of the model used in the rest of the paper. As mentioned in Sec. I the model consists of three generations of sequential leptons $(e, \mu, \tau)$ and in addition a single vectorlike generation. Thus one has four sequential families and a mirror generation. The properties of the sequential generation under $S U(3)_{C} \times S U(2)_{L} \times$ $U(1)_{Y}$ are given by

$$
\begin{aligned}
\psi_{i L} & \equiv\left(\begin{array}{c}
\nu_{i L} \\
\ell_{i L}
\end{array}\right) \sim\left(1,2,-\frac{1}{2}\right), \\
\ell_{i L}^{c} & \sim(1,1,1), \quad \nu_{i L}^{c} \sim(1,1,0),
\end{aligned}
$$

where the last entry on the right-hand side of each $\sim$ is the value of the hypercharge $Y$ defined so that $Q=T_{3}+Y$ and we have included in our analysis the singlet field $\nu_{i}^{c}$, where $i$ runs from $1-4$. The mirrors are given by

$$
\begin{aligned}
\chi^{c} & \equiv\left(\begin{array}{c}
E_{\mu L}^{c} \\
N_{L}^{c}
\end{array}\right) \sim\left(1,2, \frac{1}{2}\right), \\
E_{\mu L} & \sim(1,1,-1), \quad N_{L} \sim(1,1,0) .
\end{aligned}
$$

The main difference between the leptons and the mirrors is that while the leptons have $V-A$ type interactions with $S U(2)_{L} \times U(1)_{Y}$ gauge bosons the mirrors have $V+A$ type interactions.

We assume that the mirrors of the vectorlike generation escape acquiring mass at the grand unified theory (GUT) scale and remain light down to the electroweak scale where the superpotential of the model for the lepton part may be written in the form

$$
\begin{aligned}
W= & -\mu \epsilon_{i j} \hat{H}_{1}^{i} \hat{H}_{2}^{j}+\epsilon_{i j}\left[f_{1} \hat{H}_{1}^{i} \hat{\psi}_{L}^{j} \hat{\tau}_{L}^{c}+f_{1}^{\prime} \hat{H}_{2}^{j} \hat{\psi}_{L}^{i} \hat{\nu}_{\tau L}^{c}+f_{2} \hat{H}_{1}^{i} \hat{\chi}^{c j} \hat{N}_{L}+f_{2}^{\prime} \hat{H}_{2}^{j} \hat{\chi}^{c i} \hat{E}_{L}\right. \\
& \left.+h_{1} \hat{H}_{1}^{i} \hat{\psi}_{\mu L}^{j} \hat{\mu}_{L}^{c}+h_{1}^{\prime} \hat{H}_{2}^{j} \hat{\psi}_{\mu L}^{i} \hat{\nu}_{\mu L}^{c}+h_{2} \hat{H}_{1}^{i} \hat{\psi}_{e L}^{j} \hat{e}_{L}^{c}+h_{2}^{\prime} \hat{H}_{2}^{j} \hat{\psi}_{e L}^{i} \hat{\nu}_{e L}^{c}+y_{5} \hat{H}_{1}^{i} \hat{\psi}_{4 L}^{j} \hat{\ell}_{4 L}^{c}+y_{5}^{\prime} \hat{H}_{2}^{j} \hat{\psi}_{4 L}^{i} \hat{\nu}_{4 L}^{c}\right] \\
& +f_{3} \epsilon_{i j} \hat{\chi}^{c i} \hat{\psi}_{L}^{j}+f_{3}^{\prime} \epsilon_{i j} \hat{\chi}^{c i} \hat{\psi}_{\mu L}^{j}+f_{4} \hat{\tau}_{L}^{c} \hat{E}_{L}+f_{5} \hat{\nu}_{\tau L}^{c} \hat{N}_{L}+f_{4}^{\prime} \hat{\mu}_{L}^{c} \hat{E}_{L}+f_{5}^{\prime} \hat{\nu}_{\mu L}^{c} \hat{N}_{L} \\
& +f_{3}^{\prime \prime} \epsilon_{i j} \hat{\chi}^{c i} \hat{\psi}_{e L}^{j}+f_{4}^{\prime \prime} \hat{e}_{L}^{c} \hat{E}_{L}+f_{5}^{\prime \prime} \hat{\nu}_{e L}^{c} \hat{N}_{L}+h_{6} \epsilon_{i j} \hat{\chi}^{c i} \hat{\psi}_{4 L}^{j}+h_{7} \hat{\ell}_{4 L}^{c} \hat{E}_{L}+h_{8} \hat{\nu}_{4 L}^{c} \hat{N}_{L},
\end{aligned}
$$

where ^implies superfields, $\hat{\psi}_{L} \equiv \hat{\psi}_{\tau L}$ stands for $\hat{\psi}_{3 L}, \hat{\psi}_{\mu L}$ stands for $\hat{\psi}_{2 L}$, and $\hat{\psi}_{e L}$ stands for $\hat{\psi}_{1 L}$.

The mass terms for the neutrinos, mirror neutrinos, leptons and mirror leptons arise from the term

$$
\mathcal{L}=-\frac{1}{2} \frac{\partial^{2} W}{\partial A_{i} \partial A_{j}} \psi_{i} \psi_{j}+\text { H.c. },
$$

where $\psi$ and $A$ stand for generic two-component fermion and scalar fields. After spontaneous breaking of the electroweak symmetry, $\left(\left\langle H_{1}^{1}\right\rangle=v_{1} / \sqrt{2}\right.$ and $\left.\left\langle H_{2}^{2}\right\rangle=v_{2} / \sqrt{2}\right)$, we have the following set of mass terms written in the fourcomponent spinor notation so that

$$
-\mathcal{L}_{m}=\bar{\xi}_{R}^{T}\left(M_{f}\right) \xi_{L}+\bar{\eta}_{R}^{T}\left(M_{\ell}\right) \eta_{L}+\text { H.c. },
$$

where the basis vectors in which the mass matrix is written are given by

$$
\begin{aligned}
& \bar{\xi}_{R}^{T}=\left(\bar{\nu}_{\tau R} \bar{N}_{R} \bar{\nu}_{\mu R} \bar{\nu}_{e R} \bar{\nu}_{4 R}\right), \\
& \xi_{L}^{T}=\left(\nu_{\tau L} N_{L} \nu_{\mu L} \nu_{e L} \nu_{4 L}\right), \\
& \bar{\eta}_{R}^{T}=\left(\overline{\tau_{R}} \bar{E}_{R} \overline{\mu_{R}} \overline{e_{R}} \bar{\ell}_{4 R}\right), \\
& \eta_{L}^{T}=\left(\tau_{L} E_{L} \mu_{L} e_{L} \ell_{4 L}\right),
\end{aligned}
$$

and the mass matrix $M_{f}$ of neutrinos is given by

$$
M_{f}=\left(\begin{array}{ccccc}
f_{1}^{\prime} v_{2} / \sqrt{2} & f_{5} & 0 & 0 & 0 \\
-f_{3} & f_{2} v_{1} / \sqrt{2} & -f_{3}^{\prime} & -f_{3}^{\prime \prime} & -h_{6} \\
0 & f_{5}^{\prime} & h_{1}^{\prime} v_{2} / \sqrt{2} & 0 & 0 \\
0 & f_{5}^{\prime \prime} & 0 & h_{2}^{\prime} v_{2} / \sqrt{2} & 0 \\
0 & h_{8} & 0 & 0 & y_{5}^{\prime} v_{2} / \sqrt{2}
\end{array}\right) .
$$

We define the matrix elements $(2,2)$ and $(5,5)$ of the mass matrix as $m_{N}$ and $m_{G}^{\nu}$, respectively, so that

$$
m_{N}=f_{2} v_{1} / \sqrt{2} \text { and } m_{G}^{\nu}=y_{5}^{\prime} v_{2} / \sqrt{2} .
$$


The mass matrix is not Hermitian and thus one needs biunitary transformations to diagonalize it. We define the biunitary transformation so that

$$
D_{R}^{\nu \dagger}\left(M_{f}\right) D_{L}^{\nu}=\operatorname{diag}\left(m_{\psi_{1}}, m_{\psi_{2}}, m_{\psi_{3}}, m_{\psi_{4}}, m_{\psi_{5}}\right),
$$

where $\psi_{1}, \psi_{2}, \psi_{3}, \psi_{4}, \psi_{5}$ are the mass eigenstates for the neutrinos. In the limit of no mixing we identify $\psi_{1}$ as the light tau neutrino, $\psi_{2}$ as the heavier mass mirror eigenstate, $\psi_{3}$ as the muon neutrino, $\psi_{4}$ as the electron neutrino and $\psi_{5}$ as the other heavy four-sequential generation neutrino. A similar analysis goes to the lepton mass matrix $M_{\ell}$ where

$$
M_{\ell}=\left(\begin{array}{ccccc}
f_{1} v_{1} / \sqrt{2} & f_{4} & 0 & 0 & 0 \\
f_{3} & f_{2}^{\prime} v_{2} / \sqrt{2} & f_{3}^{\prime} & f_{3}^{\prime \prime} & h_{6} \\
0 & f_{4}^{\prime} & h_{1} v_{1} / \sqrt{2} & 0 & 0 \\
0 & f_{4}^{\prime \prime} & 0 & h_{2} v_{1} / \sqrt{2} & 0 \\
0 & h_{7} & 0 & 0 & y_{5} v_{1} / \sqrt{2}
\end{array}\right) .
$$

We introduce now the mass parameters $m_{E}$ and $m_{G}$ for the elements $(2,2)$ and $(5,5)$, respectively, of the mass matrix above so that

$$
m_{E}=f_{2}^{\prime} v_{2} / \sqrt{2} \text { and } m_{G}=y_{5} v_{1} / \sqrt{2} .
$$

$C P$ phases that arise from the new sector are defined so that

$$
\begin{aligned}
f_{i} & =\left|f_{i}\right| e^{i \chi_{i}}, \quad f_{i}^{\prime}=\left|f_{i}^{\prime}\right| e^{i \chi_{i}^{\prime}}, \\
f_{i}^{\prime \prime} & =\left|f_{i}^{\prime \prime}\right| e^{i \chi_{i}^{\prime \prime}} \quad(i=3,4,5), \\
h_{k} & =\left|h_{k}\right| e^{i \chi_{k}}, \quad k=6,7,8 .
\end{aligned}
$$

As in the neutrino mass matrix case, the charged lepton mass matrix is not Hermitian and thus one needs again a biunitary transformation to diagonalize it. We define the biunitary transformation so that

$$
\begin{aligned}
-\mathcal{L}_{D}= & \frac{1}{2} m_{Z}^{2} \cos ^{2} \theta_{W} \cos 2 \beta\left\{\tilde{\nu}_{\tau L} \tilde{\nu}_{\tau L}^{*}-\tilde{\tau}_{L} \tilde{\tau}_{L}^{*}+\tilde{\nu}_{\mu L} \tilde{\nu}_{\mu L}^{*}-\tilde{\mu}_{L} \tilde{\mu}_{L}^{*}+\tilde{\nu}_{e L} \tilde{\nu}_{e L}^{*}-\tilde{e}_{L} \tilde{e}_{L}^{*}+\tilde{E}_{R} \tilde{E}_{R}^{*}-\tilde{N}_{R} \tilde{N}_{R}^{*}+\tilde{\nu}_{4 L} \tilde{\tau}_{4 L}^{*}-\tilde{\ell}_{4 L} \tilde{\ell}_{4 L}^{*}\right\} \\
& +\frac{1}{2} m_{Z}^{2} \sin ^{2} \theta_{W} \cos 2 \beta\left\{\tilde{\nu}_{\tau L} \tilde{\nu}_{\tau L}^{*}+\tilde{\tau}_{L} \tilde{\tau}_{L}^{*}+\tilde{\nu}_{\mu L} \tilde{\nu}_{\mu L}^{*}+\tilde{\mu}_{L} \tilde{\mu}_{L}^{*}+\tilde{\nu}_{e L} \tilde{\nu}_{e L}^{*}+\tilde{e}_{L} \tilde{e}_{L}^{*}+\tilde{\nu}_{4 L} \tilde{L}_{4 L}^{*}+\tilde{\ell}_{4 L} \tilde{\ell}_{4 L}^{*}\right. \\
& \left.-\tilde{E}_{R} \tilde{E}_{R}^{*}-\tilde{N}_{R} \tilde{N}_{R}^{*}+2 \tilde{E}_{L} \tilde{E}_{L}^{*}-2 \tilde{\tau}_{R} \tilde{\tau}_{R}^{*}-2 \tilde{\mu}_{R} \tilde{\mu}_{R}^{*}-2 \tilde{e}_{R} \tilde{e}_{R}^{*}-2 \tilde{\ell}_{4 R} \tilde{\ell}_{4 R}^{*}\right\}
\end{aligned}
$$

and $\mathcal{L}_{\text {soft }}$ is given in Appendix A.

\section{THE ANALYSIS OF $\mathcal{B}\left(\boldsymbol{\ell}_{i} \rightarrow \boldsymbol{\ell}_{j} \gamma\right)$ WITH INCLUSION OF VECTORLIKE LEPTONS}

Stringent bounds exist on the decay $\mu \rightarrow e \gamma$ from the MEG experiment [1]:

$\mathcal{B}(\mu \rightarrow e \gamma)<5.7 \times 10^{-13}$ at $90 \%$ C.L. $\quad(\mathrm{MEG})$.

Other flavor changing decays are $\tau \rightarrow \mu \gamma$ and $\tau \rightarrow e \gamma$. Here the current experimental limits on the branching ratios of these processes from the $B A B A R$ collaboration [2] and from the Belle collaboration [3] are

$$
\begin{aligned}
& \mathcal{B}(\tau \rightarrow \mu \gamma)<4.4 \times 10^{-8} \text { at } 90 \% \text { C.L. }(B A B A R) \text {, } \\
& \mathcal{B}(\tau \rightarrow \mu \gamma)<4.5 \times 10^{-8} \text { at } 90 \% \text { C.L. (Belle), } \\
& \mathcal{B}(\tau \rightarrow e \gamma)<3.3 \times 10^{-8} \text { at } 90 \% \text { C.L. }(B A B A R) \text {. }
\end{aligned}
$$

Improvement in the measurements of flavor changing processes is expected to occur at the SuperB factories [28-30] (for a review see [31]). Thus it is of interest to see if 
theoretical estimates for these branching ratios can lie close to the current experimental limits to be detectable in an improved experiment. Flavor violating radiative decays have been analyzed in several previous works (see, e.g., [31-37]). However, none of these works explore the class of models discussed here.

We discuss now the specifics of the model. Thus the decay $\mu \rightarrow e \gamma$ is induced by one-loop electric and magnetic transition dipole moments, which arise from the diagrams of Fig. 1. For an incoming muon of momentum $p$ and a resulting electron of momentum $p^{\prime}$, we define the amplitude,

$$
\left\langle e\left(p^{\prime}\right)\left|J_{\alpha}\right| \mu(p)\right\rangle=\bar{u}_{e}\left(p^{\prime}\right) \Gamma_{\alpha} u_{\mu}(p)
$$

where

$$
\Gamma_{\alpha}(q)=\frac{F_{2}^{\mu e}(q) i \sigma_{\alpha \beta} q^{\beta}}{m_{\mu}+m_{e}}+\frac{F_{3}^{\mu e}(q) \sigma_{\alpha \beta} \gamma_{5} q^{\beta}}{m_{\mu}+m_{e}}+\cdots
$$

with $q=p^{\prime}-p$ and where $m_{f}$ denotes the mass of the fermion $f$. The branching ratio of $\mu \rightarrow e \gamma$ is given by

$$
\mathcal{B}(\mu \rightarrow e \gamma)=\frac{24 \pi^{2}}{G_{F}^{2} m_{\mu}^{2}\left(m_{\mu}+m_{e}\right)^{2}}\left\{\left|F_{2}^{\mu e}(0)\right|^{2}+\left|F_{3}^{\mu e}(0)\right|^{2}\right\}
$$

where the form factors $F_{2}^{\mu e}$ and $F_{3}^{\mu e}$ arise from the chargino, neutralino and vector boson contributions as follows:

$$
\begin{aligned}
& F_{2}^{\mu e}(0)=F_{2 \chi^{+}}^{\mu e}+F_{2 \chi^{0}}^{\mu e}+F_{2 W}^{\mu e}+F_{2 Z}^{\mu e}, \\
& F_{3}^{\mu e}(0)=F_{3 \chi^{+}}^{\mu e}+F_{3 \chi^{0}}^{\mu e}+F_{3 W}^{\mu e}+F_{3 Z}^{\mu e}
\end{aligned}
$$

It is also useful to define $\mathcal{B}_{m}$ and $\mathcal{B}_{e}$ as follows:
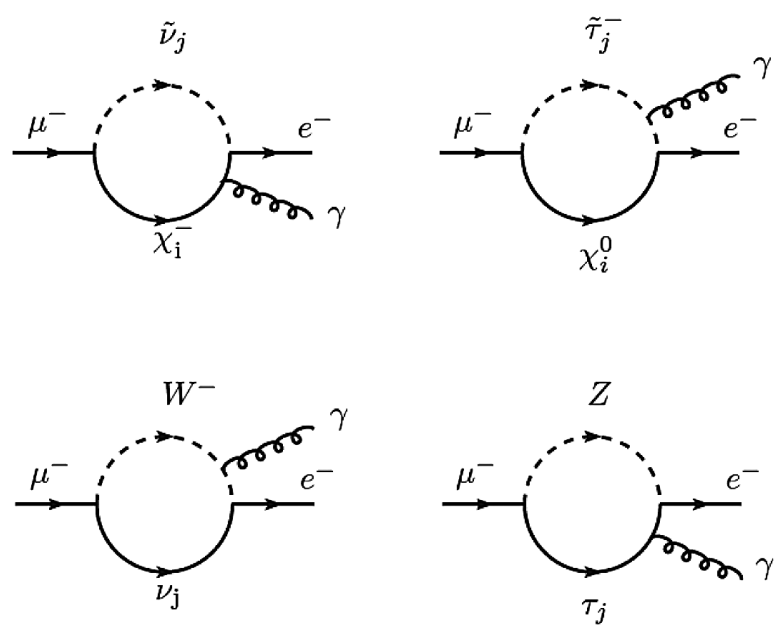

FIG. 1. The diagrams that allow the decay of $\mu \rightarrow e \gamma$ via supersymmetric loops involving the chargino (top left) and the neutralino (top right) and via $W$ loop (bottom left) and $Z$ loop (bottom right) with emission of the photon from the charged particle inside the loop.

$$
\begin{aligned}
& \mathcal{B}_{m}(\mu \rightarrow e \gamma)=\frac{24 \pi^{2}}{G_{F}^{2} m_{\mu}^{2}\left(m_{\mu}+m_{e}\right)^{2}}\left|F_{2}^{\mu e}(0)\right|^{2}, \\
& \mathcal{B}_{e}(\mu \rightarrow e \gamma)=\frac{24 \pi^{2}}{G_{F}^{2} m_{\mu}^{2}\left(m_{\mu}+m_{e}\right)^{2}}\left|F_{3}^{\mu e}(0)\right|^{2},
\end{aligned}
$$

where $\mathcal{B}_{m}$ is the branching ratio from the magnetic dipole operator and $\mathcal{B}_{e}$ is the branching ratio from the electric dipole operator. We discuss now the individual contributions to $F_{2}^{\mu e}$ and $F_{3}^{\mu e}$ from supersymmetric and nonsupersymmetric loops.

The chargino contribution $F_{2 \chi^{+}}^{\mu e}$ is given by

$$
F_{2 \chi^{+}}^{\mu e}=\sum_{i=1}^{2} \sum_{j=1}^{10}\left[\frac{-m_{\mu}\left(m_{\mu}+m_{e}\right)}{192 \pi^{2} m_{\tilde{\chi}_{i}^{+}}^{2}}\left\{C_{4 i j}^{L} C_{3 i j}^{L *}+C_{4 i j}^{R} C_{3 i j}^{R *}\right\} F_{4}\left(\frac{M_{\tilde{\nu}_{j}}^{2}}{m_{\tilde{\chi}_{i}^{+}}^{2}}\right)+\frac{\left(m_{\mu}+m_{e}\right)}{64 \pi^{2} m_{\tilde{\chi}_{i}}}\left\{C_{4 i j}^{L} C_{3 i j}^{R *}+C_{4 i j}^{R} C_{3 i j}^{L *}\right\} F_{3}\left(\frac{M_{\tilde{\nu}_{j}}^{2}}{m_{\tilde{\chi}^{+}}^{2}}\right)\right],
$$

where $F_{3}(x)$ and $F_{4}(x)$ are given by

$$
F_{3}(x)=\frac{1}{(x-1)^{3}}\left[3 x^{2}-4 x+1-2 x^{2} \ln x\right],
$$

and

$$
F_{4}(x)=\frac{1}{(x-1)^{4}}\left[2 x^{3}+3 x^{2}-6 x+1-6 x^{2} \ln x\right] .
$$

The neutralino contribution $F_{2 \chi^{0}}^{\mu e}$ is given by

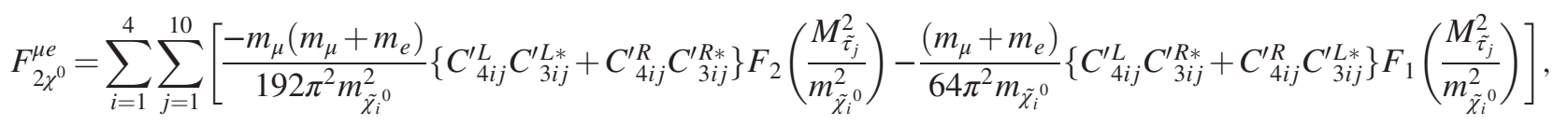


where $F_{1}(x)$ and $F_{2}(x)$ are given by

$$
F_{1}(x)=\frac{1}{(x-1)^{3}}\left[1-x^{2}+2 x \ln x\right]
$$

and

$$
F_{2}(x)=\frac{1}{(x-1)^{4}}\left[-x^{3}+6 x^{2}-3 x-2-6 x \ln x\right] .
$$

The contributions from the $W$ exchange $F_{2 W}^{\mu e}$ are given by

$$
F_{2 W}^{\mu e}=\sum_{i=1}^{5}\left[\frac{m_{\mu}\left(m_{\mu}+m_{e}\right)}{32 \pi^{2} m_{W}^{2}}\left[C_{L i 4}^{W} C_{L i 3}^{W *}+C_{R i 4}^{W} C_{R i 3}^{W *}\right] F_{W}\left(\frac{m_{\psi_{i}}^{2}}{m_{W}^{2}}\right)+\frac{m_{\psi_{i}}\left(m_{\mu}+m_{e}\right)}{32 \pi^{2} m_{W}^{2}}\left[C_{L i 4}^{W} C_{R i 3}^{W *}+C_{R i 4}^{W} C_{L i 3}^{W *}\right] G_{W}\left(\frac{m_{\psi_{i}}^{2}}{m_{W}^{2}}\right)\right],
$$

where the form factors $F_{W}(x)$ and $G_{W}(x)$ are given by

$$
F_{W}(x)=\frac{1}{6(x-1)^{4}}\left[4 x^{4}-49 x^{3}+18 x^{3} \ln x+78 x^{2}-43 x+10\right],
$$

and

$$
G_{W}(x)=\frac{1}{(x-1)^{3}}\left[4-15 x+12 x^{2}-x^{3}-6 x^{2} \ln x\right] .
$$

The contribution $F_{2 Z}^{\mu e}$ from the $Z$ exchange is given by

$F_{2 Z}^{\mu e}=\sum_{\beta=1}^{5}\left[\frac{m_{\mu}\left(m_{\mu}+m_{e}\right)}{64 \pi^{2} m_{Z}^{2}}\left[C_{L \beta 4}^{Z} C_{L \beta 3}^{Z *}+C_{R \beta 4}^{Z} C_{R \beta 3}^{Z *}\right] F_{Z}\left(\frac{m_{\tau_{\beta}}^{2}}{m_{Z}^{2}}\right)+\frac{m_{\tau_{\beta}}\left(m_{\mu}+m_{e}\right)}{64 \pi^{2} m_{Z}^{2}}\left[C_{L \beta 4}^{Z} C_{R \beta 3}^{Z *}+C_{R \beta 4}^{Z} C_{L \beta 3}^{Z *}\right] G_{Z}\left(\frac{m_{\tau_{\beta}}^{2}}{m_{Z}^{2}}\right)\right]$,

where the form factors $F_{Z}(x)$ and $G_{Z}(x)$ are given by

$$
F_{Z}(x)=\frac{1}{3(x-1)^{4}}\left[-5 x^{4}+14 x^{3}-39 x^{2}+18 x^{2} \ln x+38 x-8\right],
$$

and

$$
G_{Z}(x)=\frac{2}{(x-1)^{3}}\left[x^{3}+3 x-6 x \ln x-4\right] .
$$

The chargino contribution $F_{3 \chi^{+}}^{\mu e}$ is given by

$$
F_{3 \chi^{+}}^{\mu e}=\sum_{i=1}^{2} \sum_{j=1}^{10} \frac{\left(m_{\mu}+m_{e}\right) m_{\tilde{\chi}_{i}^{+}}}{32 \pi^{2} M_{\tilde{\nu}_{j}}^{2}}\left[C_{4 i j}^{L} C_{3 i j}^{R *}-C_{4 i j}^{R} C_{3 i j}^{L *}\right] F_{6}\left(\frac{m_{\tilde{\chi}_{i}^{+}}^{2}}{M_{\tilde{\nu}_{j}}^{2}}\right),
$$

where

$$
F_{6}(x)=\frac{1}{2(x-1)^{2}}\left[-x+3+\frac{2 \ln x}{1-x}\right] .
$$

The neutralino contribution $F_{3 \chi^{0}}^{\mu e}$ is given by

$$
F_{3 \chi^{0}}^{\mu e}=\sum_{i=1}^{4} \sum_{j=1}^{10} \frac{\left(m_{\mu}+m_{e}\right) m_{\tilde{\chi}_{i}^{0}}}{32 \pi^{2} M_{\tilde{\tau}_{j}}^{2}}\left[C_{4 i j}^{L} C_{3 i j}^{\prime R *}-C_{4 i j}^{\prime R} C_{3 i j}^{\prime L *}\right] F_{5}\left(\frac{m_{\tilde{\chi}_{i}^{0}}^{2}}{M_{\tilde{\tau}_{j}}^{2}}\right),
$$


where

$$
F_{5}(x)=\frac{1}{2(x-1)^{2}}\left[x+1+\frac{2 x \ln x}{1-x}\right]
$$

The $W$ boson contribution $F_{3 W}^{\mu e}$ is given by

$F_{3 W}^{\mu e}=-\sum_{i=1}^{5} \frac{m_{\psi_{i}}\left(m_{\mu}+m_{e}\right)}{32 \pi^{2} m_{W}^{2}}\left[C_{L i 4}^{W} C_{R i 3}^{W *}-C_{R i 4}^{W} C_{L i 3}^{W *}\right] I_{1}\left(\frac{m_{\psi_{i}}^{2}}{m_{W}^{2}}\right)$

where the form factor $I_{1}$ is given by

$$
I_{1}(x)=\frac{2}{(1-x)^{2}}\left[1-\frac{11}{4} x+\frac{1}{4} x^{2}-\frac{3 x^{2} \ln x}{2(1-x)}\right]
$$

Finally, the $Z$ exchange diagram contribution $F_{3 Z}^{\mu e}$ is given by

$F_{3 Z}^{\mu e}=\sum_{\beta=1}^{5} \frac{\left(m_{\mu}+m_{e}\right)}{32 \pi^{2}} \frac{m_{\tau_{\beta}}}{m_{Z}^{2}}\left[C_{L 4 \beta}^{Z} C_{R 3 \beta}^{Z *}-C_{R 4 \beta}^{Z} C_{L 3 \beta}^{Z *}\right] I_{2}\left(\frac{m_{\tau_{\beta}}^{2}}{m_{Z}^{2}}\right)$,

where the form factor $I_{2}$ is given by

$$
I_{2}(x)=\frac{2}{(1-x)^{2}}\left[1+\frac{1}{4} x+\frac{1}{4} x^{2}+\frac{3 x \ln x}{2(1-x)}\right]
$$

All couplings $C^{L}, C^{R}, C^{L}, C^{\prime R}, C_{L}^{W}, C_{R}^{W}, C_{L}^{Z}$ and $C_{R}^{Z}$ in Eqs. (25)-(37), (39), (41) and (43), are given in Appendix B.

An analysis for $\mathcal{B}(\tau \rightarrow e \gamma)$ can be done similarly so that

$\mathcal{B}(\tau \rightarrow e \gamma)=\frac{24 \pi^{2}}{G_{F}^{2} m_{\tau}^{2}\left(m_{\tau}+m_{e}\right)^{2}}\left\{\left|F_{2}^{\tau e}(0)\right|^{2}+\left|F_{3}^{\tau e}(0)\right|^{2}\right\}$,

where the expressions for the form factors, $F_{2}^{\tau e}$ and $F_{3}^{\tau e}$, can be obtained from Eqs. (21) and (22) by the replacements: $m_{\mu} \rightarrow m_{\tau}$ and $C_{3 i j}, C_{3 i j}^{\prime}, C_{i 3}^{W}, C_{\beta 3}^{Z} \rightarrow C_{1 i j}, C_{1 i j}^{\prime}, C_{i 1}^{W}, C_{\beta 1}^{Z}$.

Also for $\mathcal{B}(\tau \rightarrow \mu \gamma)$ we have

$\mathcal{B}(\tau \rightarrow \mu \gamma)=\frac{24 \pi^{2}}{G_{F}^{2} m_{\tau}^{2}\left(m_{\tau}+m_{\mu}\right)^{2}}\left\{\left|F_{2}^{\tau \mu}(0)\right|^{2}+\left|F_{3}^{\tau \mu}(0)\right|^{2}\right\}$,

where the expressions for the form factors $F_{2}^{\tau \mu}$ and $F_{3}^{\tau \mu}$ can be deduced from Eqs. (21) and (22) by the replacements: $m_{\mu} \rightarrow m_{\tau}, m_{e} \rightarrow m_{\mu}, C_{3 i j}, C_{3 i j}^{\prime}, C_{i 3}^{W}, C_{\beta 3}^{Z} \rightarrow C_{1 i j}, C_{1 i j}^{\prime}, C_{i 1}^{W}$, $C_{\beta 1}^{Z}$ and $C_{4 i j}, C_{4 i j}^{\prime}, C_{i 4}^{W}, C_{\beta 4}^{Z} \rightarrow C_{3 i j}, C_{3 i j}^{\prime}, C_{i 3}^{W}, C_{\beta 3}^{Z}$.

\section{EDM ANALYSIS BY INCLUSION OF VECTORLIKE LEPTONS}

The electric dipole moment (EDM) of elementary particles arises only at the multiloop level in the standard model and is beyond the scope of observation in the current or in the near future experiment. However, beyond the standard model physics can generate EDMs which are within the range of observability. The current experimental limits on $d_{e}, d_{\mu}, d_{\tau}$ are as follows. For the electron we have [4]

$$
d_{e}<9.3 \times 10^{-29} e \mathrm{~cm} \quad(90 \% \text { C.L. })
$$

For the muon the current limit on the EDM is [6]

$$
d_{\mu}<1.9 \times 10^{-19} e \mathrm{~cm} \quad(95 \% \text { C.L. }) .
$$

The current experimental limit on the EDM of the tau lepton is [38]

$$
d_{\tau}<1.1 \times 10^{-17} e \mathrm{~cm}
$$

Next we discuss the case when we include a vectorlike leptonic multiplet which mixes with the three generations of leptons. In this case the mass eigenstates will be linear combinations of the three generations plus the vectorlike generation which includes mirror particles. Here we discuss the contribution of the model to the lepton EDM. These contributions arise from four sources: the chargino exchange, the neutralino exchange, the $W$ boson exchange and the $Z$ boson exchange as shown in Fig. 2 .
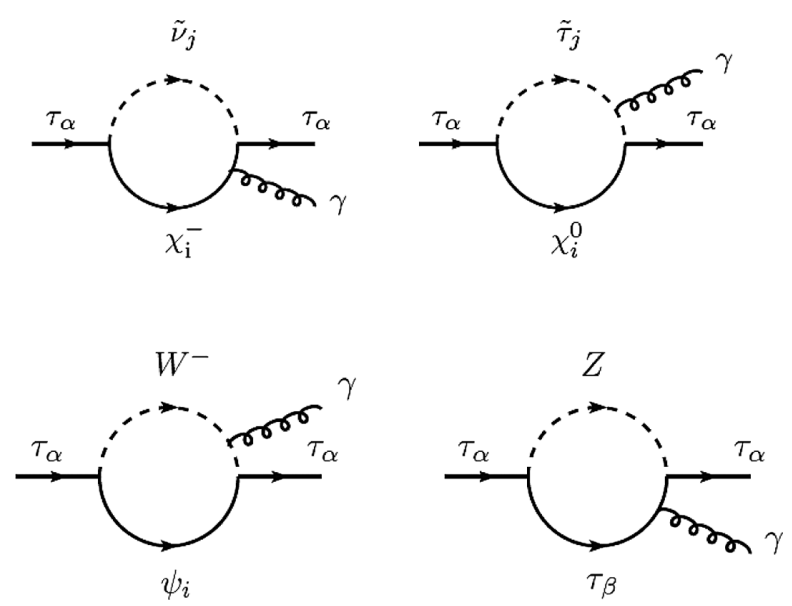

FIG. 2. Upper diagrams: Supersymmetric contributions to the leptonic EDMs arising from the exchange of the charginos, sneutrinos and mirror sneutrinos (upper left) and the exchange of neutralinos, sleptons, and mirror sleptons (upper right) inside the loop. Lower diagrams: Nonsupersymmetric diagrams that contribute to the leptonic EDMs via the exchange of the $W$, the sequential and vectorlike neutrinos (lower left) and the exchange of the $Z$, the sequential and vectorlike charged leptons (lower right). 
Using the interactions given in Appendix B, the chargino contribution is given by

$d_{\alpha}^{\chi^{+}}=-\frac{1}{16 \pi^{2}} \sum_{i=1}^{2} \sum_{j=1}^{10} \frac{m_{\chi_{i}^{+}}}{m_{\tilde{\nu}_{j}}^{2}} \operatorname{Im}\left(C_{\alpha i j}^{L} C_{\alpha i j}^{R *}\right) F_{6}\left(\frac{m_{\chi_{i}^{+}}^{2}}{m_{\tilde{\nu}_{j}}^{2}}\right)$,

where the form factor $F_{6}(x)$ is given by Eq. (38).

Using the interactions given in Appendix $\mathrm{B}$, the neutralino contribution is given by

$d_{\alpha}^{\chi^{0}}=-\frac{1}{16 \pi^{2}} \sum_{i=1}^{4} \sum_{j=1}^{10} \frac{m_{\chi_{i}^{0}}}{m_{\tilde{\tau}_{j}}^{2}} \operatorname{Im}\left(C_{\alpha i j}^{\prime L} C_{\alpha i j}^{\prime R *}\right) F_{5}\left(\frac{m_{\chi_{i}^{0}}^{2}}{m_{\tilde{\tau}_{j}}^{2}}\right)$,

where the form factor $F_{5}(x)$ is given by Eq. (40).

The contributions to the lepton electric dipole moment from the $W$ and $Z$ exchange arise from similar loops. Using the interactions given in Appendix B the contribution arising from the $W$ exchange diagram is given by

$$
d_{\alpha}^{W}=\frac{1}{16 \pi^{2}} \sum_{i=1}^{5} \frac{m_{\psi_{i}^{+}}}{m_{W}^{2}} \operatorname{Im}\left(C_{L i \alpha}^{W} C_{R i \alpha}^{W *}\right) I_{1}\left(\frac{m_{\psi_{i}}^{2}}{m_{W}^{2}}\right),
$$

where the form factor $I_{1}$ is given by Eq. (42).

The $Z$ boson exchange diagram contribution is given by

$$
d_{\alpha}^{Z}=-\frac{1}{16 \pi^{2}} \sum_{\beta=1}^{5} \frac{m_{\tau_{\beta}}}{m_{Z}^{2}} \operatorname{Im}\left(C_{L \alpha \beta}^{Z} C_{R \alpha \beta}^{Z *}\right) I_{2}\left(\frac{m_{\tau_{\beta}}^{2}}{m_{Z}^{2}}\right),
$$

where the form factor $I_{2}$ is given by Eq. (44). Again, all couplings $C^{L}, C^{R}, C^{\prime L}, C^{\prime R}, C_{L}^{W}, C_{R}^{W}, C_{L}^{Z}$ and $C_{R}^{Z}$ used here are given in Appendix B.

\section{ANALYSIS OF $g-2$ WITH EXCHANGE OF VECTORLIKE LEPTONS}

The current experimental result for the muon $g-2[5]$ is

$$
\Delta a_{\mu}=a_{\mu}^{\mathrm{exp}}-a_{\mu}^{\mathrm{SM}}=(28.8 \pm 7.9) \times 10^{-10},
$$

which is about a 3 sigma deviations from the standard model prediction. For the electron $g_{e}-2$, experiment gives [39]

$$
\Delta a_{e}=a_{e}^{\mathrm{exp}}-a_{e}^{\mathrm{SM}}=8.70(8.07) \times 10^{-13} .
$$

This result relies on a QED calculation up to four loops. Thus along with Eqs. (54), (55) also acts as a constraint on the standard model extensions. We compute beyond the standard model contributions to these within the model of Sec. II. Below we discuss details of the various
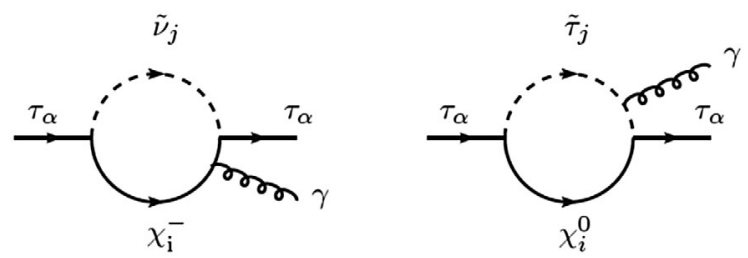

FIG. 3. The diagrams that contribute to the leptonic $\left(\tau_{\alpha}\right)$ magnetic dipole moment via exchange of charginos $\left(\chi_{i}^{-}\right)$, sneutrinos and mirror sneutrinos $\left(\tilde{\nu}_{j}\right)$ (left diagram) inside the loop and from the exchange of neutralinos $\left(\chi_{i}^{0}\right)$, sleptons and mirror sleptons $\left(\tilde{\tau}_{j}\right)$ (right diagram) inside the loop.

contributions. The contribution arising from the exchange of the charginos, sneutrinos and mirror sneutrinos as shown in the left diagram in Fig. 3 is given by

$$
\begin{aligned}
a_{\alpha}^{\chi^{+}}= & -\sum_{i=1}^{2} \sum_{j=1}^{10} \frac{m_{\tau_{\alpha}}}{16 \pi^{2} m_{\chi_{i}^{-}}} \operatorname{Re}\left(C_{\alpha i j}^{L} C_{\alpha i j}^{R *}\right) F_{3}\left(\frac{m_{\tilde{\nu}_{j}}^{2}}{m_{\chi_{i}^{-}}^{2}}\right) \\
& +\sum_{i=1}^{2} \sum_{j=1}^{10} \frac{m_{\tau_{\alpha}}^{2}}{96 \pi^{2} m_{\chi_{i}^{-}}^{2}}\left[\left|C_{\alpha i j}^{L}\right|^{2}+\left|C_{\alpha i j}^{R}\right|^{2}\right] F_{4}\left(\frac{m_{\tilde{\nu}_{j}}^{2}}{m_{\chi_{i}^{-}}^{2}}\right),
\end{aligned}
$$

where $m_{\chi_{i}^{-}}$is the mass of chargino $\chi_{i}^{-}$and $m_{\tilde{\nu}_{j}}$ is the mass of sneutrino $\tilde{\nu}_{j}$ and where the form factors $F_{3}$ and $F_{4}$ are given by Eqs. (26) and (27).

The contribution arising from the exchange of neutralinos, charged sleptons and charged mirror sleptons as shown in the right diagram of Fig. 3 is given by

$$
\begin{aligned}
a_{\alpha}^{\chi^{0}}= & \sum_{i=1}^{4} \sum_{j=1}^{10} \frac{m_{\tau_{\alpha}}}{16 \pi^{2} m_{\chi_{i}^{0}}} \operatorname{Re}\left(C_{\alpha i j}^{L L} C_{\alpha i j}^{\prime R *}\right) F_{1}\left(\frac{m_{\tilde{\tau}_{j}}^{2}}{m_{\chi_{i}^{0}}^{2}}\right) \\
& +\sum_{i=1}^{4} \sum_{j=1}^{10} \frac{m_{\tau_{\alpha}}^{2}}{96 \pi^{2} m_{\chi_{i}^{0}}^{2}}\left[\left|C_{\alpha i j}^{L}\right|^{2}+\left|C_{\alpha i j}^{\prime R}\right|^{2}\right] F_{2}\left(\frac{m_{\tilde{\tau}_{j}}^{2}}{m_{\chi_{i}^{0}}^{2}}\right),
\end{aligned}
$$

where the form factors $F_{1}$ and $F_{2}$ are given by Eqs. (29) and (30).
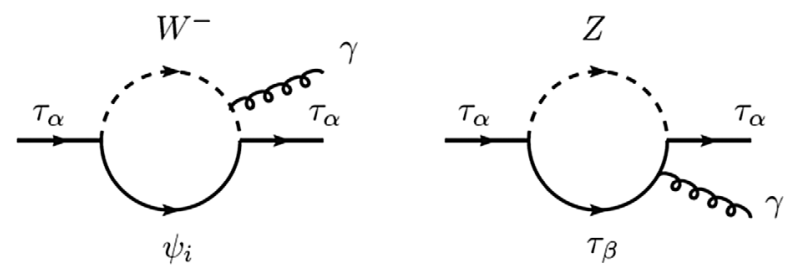

FIG. 4. The W loop (the left diagram) involving the exchange of sequential and vectorlike neutrinos $\psi_{i}$ and the $\mathrm{Z}$ loop (the right diagram) involving the exchange of sequential and vectorlike charged leptons $\tau_{\beta}$ that contribute to the magnetic dipole moment of the charged lepton $\tau_{\alpha}$. 
Next we compute the contribution from the exchange of the $W$ and $Z$ bosons. Thus the exchange of the $W$ and the exchange of neutrinos and mirror neutrinos as shown in the left diagram of Fig. 4 gives

$$
\begin{aligned}
a_{\tau_{\alpha}}^{W}= & \frac{m_{\tau_{\alpha}}^{2}}{16 \pi^{2} m_{W}^{2}} \sum_{i=1}^{5}\left[\left[\left|C_{L i \alpha}^{W}\right|^{2}+\left|C_{R i \alpha}^{W}\right|^{2}\right] F_{W}\left(\frac{m_{\psi_{i}}^{2}}{m_{W}^{2}}\right)\right. \\
& \left.+\frac{m_{\psi_{i}}}{m_{\tau_{\alpha}}} \operatorname{Re}\left(C_{L i \alpha}^{W} C_{R i \alpha}^{W *}\right) G_{W}\left(\frac{m_{\psi_{i}}^{2}}{m_{W}^{2}}\right)\right],
\end{aligned}
$$

where the form factors $F_{W}$ and $G_{W}$ are given by Eqs. (32) and (33).

Finally the exchange of the $Z$ and the exchange of leptons and mirror leptons as shown in the right diagram of Fig. 4 gives

$$
\begin{aligned}
a_{\tau_{\alpha}}^{Z}= & \frac{m_{\tau_{\alpha}}^{2}}{32 \pi^{2} m_{Z}^{2}} \sum_{\beta=1}^{5}\left[\left[\left|C_{L \beta \alpha}^{Z}\right|^{2}+\left|C_{R \beta \alpha}^{Z}\right|^{2}\right] F_{Z}\left(\frac{m_{\tau_{\beta}}^{2}}{m_{Z}^{2}}\right)\right. \\
& \left.+\frac{m_{\tau_{\beta}}}{m_{\tau_{\alpha}}} \operatorname{Re}\left(C_{L \beta \alpha}^{Z} C_{R \beta \alpha}^{Z *}\right) G_{Z}\left(\frac{m_{\tau_{\beta}}^{2}}{m_{Z}^{2}}\right)\right]
\end{aligned}
$$

where the form factors $F_{Z}$ and $G_{Z}$ are given by Eqs. (35) and (36) and $m_{Z}$ is the $Z$ boson mass. The couplings that enter in Eqs. (56)-(59) are given in Appendix B. For other works relating the muon anomalous magnetic moment to new physics see $[40,41]$.

\section{LEPTONIC VECTORLIKE CONTRIBUTION TO $h \rightarrow \gamma \gamma$}

The observed diphoton decay of the Higgs boson shows an agreement with the standard model prediction within the limits of uncertainty which is still significant. As more data is collected and uncertainties better modeled, the signal strength, $R_{\gamma \gamma}$, will be measured with a larger accuracy and any new physics manifest as particles in the loop will be better probed. Thus the ATLAS and CMS collaborations [42,43] report a signal strength of

$$
\begin{aligned}
R_{\gamma \gamma} & \equiv \frac{\sigma(p p \rightarrow h)_{\mathrm{obs}}}{\sigma(p p \rightarrow h)_{\mathrm{SM}}} \cdot \frac{\Gamma(h \rightarrow \gamma \gamma)_{\mathrm{obs}}}{\Gamma(h \rightarrow \gamma \gamma)_{\mathrm{SM}}} \\
& =0.99_{-0.14}^{+0.15} \quad(\text { ATLAS }), \quad 1.18_{-0.14}^{+0.17} \quad(\mathrm{CMS})
\end{aligned}
$$

In the SM, the largest contribution to $h \rightarrow \gamma \gamma$ comes from the exchange of $W$ bosons and top quarks in the loop.
Thus the SM decay width of a Higgs boson of mass $m_{h}$ may be approximated by the expression [44]

$$
\begin{aligned}
\Gamma_{\mathrm{SM}}(h \rightarrow \gamma \gamma) & \approx \frac{\alpha_{\mathrm{em}}^{2} m_{h}^{3}}{256 v^{2} \pi^{3}}\left|A_{1}\left(\tau_{W}\right)+N_{c} Q_{t}^{2} A_{\frac{1}{2}}\left(\tau_{t}\right)\right|^{2} \\
& \rightarrow \frac{\alpha_{\mathrm{em}}^{2} m_{h}^{3}}{256 v^{2} \pi^{3}}\left|\mathcal{A}_{\mathrm{SM}}\right|^{2},
\end{aligned}
$$

where $\mathcal{A}_{\mathrm{SM}} \approx-6.49, A_{1}$ and $A_{\frac{1}{2}}$ are loop functions (see the Appendix of [44]), $\tau_{i}=4 m_{i}^{2} / m_{h}^{2}, N_{c}$ is the color number and $Q_{t}$ the top quark charge. The inclusion of SUSY allows for the exchange of heavier particles in the loop. In general the decay width of $h \rightarrow \gamma \gamma$ in supersymmetry takes the form

$$
\begin{aligned}
& \Gamma_{\mathrm{SUSY}}(h \rightarrow \gamma \gamma) \\
& \approx \\
& \approx \frac{\alpha_{\mathrm{em}}^{2} m_{h}^{3}}{256 v^{2} \pi^{3}} \mid \sin (\beta-\alpha) Q_{W}^{2} A_{1}\left(\tau_{W}\right)+\frac{\cos \alpha}{\sin \beta} N_{t} Q_{t}^{2} A_{\frac{1}{2}}\left(\tau_{t}\right) \\
& \quad+\frac{b_{\frac{1}{2}} v}{2} N_{f} Q_{f}^{2}\left(\cos \alpha \frac{\partial}{\partial v_{2}} \log m_{f}^{2}-\sin \alpha \frac{\partial}{\partial v_{1}} \log m_{f}^{2}\right) \\
& \quad+\left.\frac{b_{0} v}{2} N_{c, S} Q_{S}^{2}\left(\cos \alpha \frac{\partial}{\partial v_{2}} \log m_{S}^{2}-\sin \alpha \frac{\partial}{\partial v_{1}} \log m_{S}^{2}\right)\right|^{2},
\end{aligned}
$$

where $\alpha$ is the $C P$-even Higgs mixing angle, $Q_{W}$ is the $W$-boson charge, $b_{\frac{1}{2}}=\frac{4}{3}$ (for Dirac fermions of mass $m_{f}$, number $N_{f}$ and charge $Q_{f}$ ) and $b_{0}=\frac{1}{3}$ (for charged scalars of mass $m_{S}$, number $N_{c, S}$ and charge $Q_{S}$ ).

The inclusion of the vectorlike leptonic generation contributes to the fermionic and scalar parts where the latter is due to the supersymmetric partners of the vectorlike leptons.

In this analysis the couplings of the Higgs boson to the first three generations are assumed negligible in comparison with the vectorlike counterparts. Hence, the mixings between the vectorlike generation and the first three generations in Eq. (7) can be assumed negligible and so the lepton mass matrix from the vectorlike generation may be written as

$$
M_{f}^{v}=\left(\begin{array}{cc}
f_{2}^{\prime} v_{2} / \sqrt{2} & h_{6} \\
h_{7} & y_{5} v_{1} / \sqrt{2}
\end{array}\right) .
$$

The two mass-squared eigenvalues resulting from diagonalizing the matrix of Eq. (63) are 


$$
m_{1,2}^{2}=\frac{1}{4}\left[2\left|h_{6}\right|^{2}+2\left|h_{7}\right|^{2}+y_{5}^{2} v_{1}^{2}+f_{2}^{\prime 2} v_{2}^{2} \pm \sqrt{\left(2\left|h_{6}\right|^{2}+2\left|h_{7}\right|^{2}+y_{5}^{2} v_{1}^{2}+f_{2}^{\prime 2} v_{2}^{2}\right)^{2}-4\left|2 h_{6} h_{7}-f_{2}^{\prime} y_{5} v_{1} v_{2}\right|^{2}}\right]
$$

Calculating the vectorlike fermionic contribution, one finds that

$$
\sum_{i}\left[\cos \alpha \frac{\partial}{\partial v_{2}} \log m_{i}^{2}-\sin \alpha \frac{\partial}{\partial v_{1}} \log m_{i}^{2}\right]=-\frac{f_{2}^{\prime} y_{5} v}{m_{1} m_{2}} \cos (\alpha+\beta)
$$

Considering only this fermionic contribution, we find that the Higgs diphoton rate is enhanced by a factor of

$$
\begin{aligned}
\frac{\Gamma(h \rightarrow \gamma \gamma)}{\Gamma(h \rightarrow \gamma \gamma)_{\mathrm{SM}}} & \approx\left|1+\frac{1}{\mathcal{A}_{\mathrm{SM}}} b_{\frac{1}{2}} N_{f} Q_{f}^{2} \frac{-v^{2} f_{2}^{\prime} y_{5}}{2 m_{1} m_{2}} \cos (\alpha+\beta)\right|^{2} \\
& \approx\left|1+0.1 N_{f} \frac{v^{2} f_{2}^{\prime} y_{5}}{m_{1} m_{2}} \cos (\alpha+\beta)\right|^{2} \equiv\left|1+r_{f}\right|^{2}
\end{aligned}
$$

Now turning to the bosonic contribution which is due to the four scalar superpartners of the vectorlike leptons. The mass eigenvalues are obtained from a $4 \times 4$ mass-squared mixing matrix and in the basis $\left(\tilde{E}_{L}, \tilde{E}_{R}, \tilde{\ell}_{4 L}, \tilde{\ell}_{4 R}\right)$ is given by

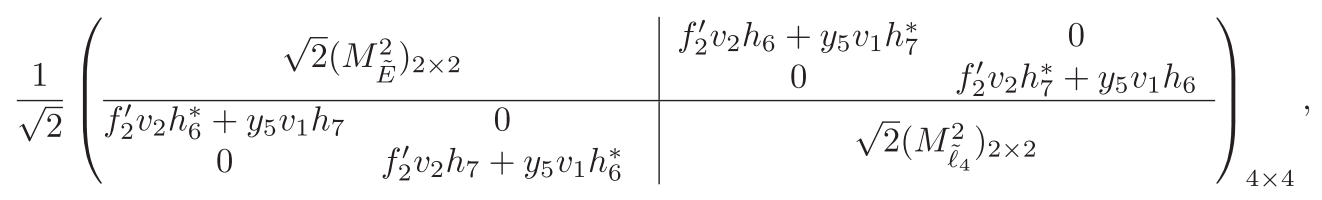

where $\left(M_{\tilde{\ell}_{4}}^{2}\right)_{2 \times 2}$ is given by

$$
\left(M_{\tilde{\ell}_{4}}^{2}\right)_{2 \times 2}=\left(\begin{array}{cc}
\tilde{M}_{4 L}^{2}+\frac{v_{1}^{2}\left|y_{5}\right|^{2}}{2}+\left|h_{6}\right|^{2}-m_{Z}^{2} \cos 2 \beta\left(\frac{1}{2}-\sin ^{2} \theta_{W}\right) & \frac{1}{\sqrt{2}} y_{5}\left(A_{4 \ell}^{*} v_{1}-\mu v_{2}\right) \\
\frac{1}{\sqrt{2}} y_{5}\left(A_{4 \ell} v_{1}-\mu^{*} v_{2}\right) & \tilde{M}_{4}^{2}+\frac{v_{1}^{2}\left|y_{5}\right|^{2}}{2}+\left|h_{7}\right|^{2}-m_{Z}^{2} \cos 2 \beta \sin ^{2} \theta_{W}
\end{array}\right),
$$

and $\left(M_{\tilde{E}}^{2}\right)_{2 \times 2}$ is given by

$$
\left(M_{\tilde{E}}^{2}\right)_{2 \times 2}=\left(\begin{array}{cc}
\tilde{M}_{\chi}^{2}+\frac{v_{2}^{2}\left|f_{2}^{\prime}\right|^{2}}{2}+\left|h_{6}\right|^{2}+m_{Z}^{2} \cos 2 \beta\left(\frac{1}{2}-\sin ^{2} \theta_{W}\right) & \frac{1}{\sqrt{2}} f_{2}^{\prime}\left(A_{E}^{*} v_{2}-\mu v_{1}\right) \\
\frac{1}{\sqrt{2}} f_{2}^{\prime}\left(A_{E} v_{2}-\mu^{*} v_{1}\right) & \tilde{M}_{E}^{2}+\frac{v_{2}^{2}\left|f_{2}^{\prime}\right|^{2}}{2}+\left|h_{7}\right|^{2}+m_{Z}^{2} \cos 2 \beta \sin ^{2} \theta_{W}
\end{array}\right) .
$$

In this analysis, the scalar masses squared, $\tilde{M}_{4 L}^{2}, \tilde{M}_{4}^{2}, \tilde{M}_{\chi}^{2}, \tilde{M}_{E}^{2}$, are much larger than the vectorlike masses, $\left|h_{6}\right|,\left|h_{7}\right|$ and so the $4 \times 4$ mass-squared matrix becomes block diagonal. Thus the two mass-squared matrices are now decoupled with superpartner $\tilde{\ell}_{4_{1,2}}$ for the first and $\tilde{E}_{1,2}$ for the second. The total bosonic contribution is the sum of the contributions coming from the two decoupled mass-squared matrices and can be written as

$$
r_{b}=r_{1}+r_{2} \equiv \frac{1}{\mathcal{A}_{\mathrm{SM}}} \frac{b_{0} v}{2} Q_{S}^{2}\left(\Sigma_{1}+\Sigma_{2}\right)
$$

Here

$$
\Sigma_{1}=\frac{1}{m_{\tilde{\ell}_{4_{1}}}^{2} m_{\tilde{\ell}_{4_{2}}}^{2}}\left[\left(2 \sin ^{2} \theta_{W} M_{11}^{2}+\cos 2 \theta_{W} M_{22}^{2}\right) \frac{m_{Z}^{2}}{v} \sin (\alpha+\beta)+\sqrt{2} M_{12}^{2} y_{5}\left(A_{4 L} \sin \alpha+\mu \cos \alpha\right)\right],
$$


and

$$
\Sigma_{2}=\frac{1}{m_{\tilde{E}_{1}}^{2} m_{\tilde{E}_{2}}^{2}}\left[\left(-2 \sin ^{2} \theta_{W} M_{11}^{\prime 2}-\cos 2 \theta_{W} M_{22}^{\prime 2}\right) \frac{m_{Z}^{2}}{v} \sin (\alpha+\beta)-\sqrt{2} M_{12}^{\prime 2} f_{2}^{\prime}\left(A_{E} \cos \alpha+\mu \sin \alpha\right)\right]
$$

where, for convenience, we renamed the matrices as $M^{2} \equiv M_{\tilde{\ell}_{4}}^{2}$ and $M^{\prime 2} \equiv M_{\tilde{E}}^{2}$. Assuming $\sigma(p p \rightarrow h)_{\mathrm{obs}}=$ $\sigma(p p \rightarrow h)_{\mathrm{SM}}$ the enhancement factor $R_{\gamma \gamma}$ is given by

$$
R_{\gamma \gamma}=\left|1+r_{f}+r_{b}\right|^{2} .
$$

\section{NUMERICAL ANALYSIS}

Here we present a correlated analysis of the observables discussed in the previous sections including the effect of vectorlike leptons (for other works related to vectorlike leptons see $[45,46])$. In the analysis we will include the $C P$ violating phases from the vectorlike generation. SUSY $C P$ phases are known to affect electroweak phenomena and these effects can be very significant [47-56]. In the analysis

TABLE I. Input parameters for the benchmark points used in this analysis along with the calculated Higgs boson $\left(h^{0}\right)$ mass. The high scale boundary conditions are obtained in the nonuniversal gaugino sector. All masses are in GeV.

\begin{tabular}{lccccccc}
\hline \hline Model & $m_{0}$ & $A_{0}$ & $m_{1}$ & $m_{2}$ & $m_{3}$ & $\tan \beta$ & $h^{0}$ \\
\hline (a) & 3974 & -10412 & 486 & 388 & 4517 & 39 & 124.5 \\
(b) & 4769 & -14593 & 463 & 245 & 3389 & 23 & 123.5 \\
(c) & 9026 & -20940 & 484 & 280 & 4143 & 14 & 124.3 \\
(d) & 3306 & -9554 & 351 & 228 & 2799 & 25 & 123.7 \\
(e) & 7004 & -8825 & 619 & 427 & 5194 & 31 & 123.5 \\
\hline \hline
\end{tabular}

we use the SUGRA model [57] with nonuniversal soft parameters given by $m_{0}, A_{0}, m_{1}, m_{2}, m_{3}, \tan \beta, \operatorname{sgn}(\mu)$, with $m_{0}$ the universal scalar mass, $A_{0}$ the universal trilinear coupling, $m_{1}, m_{2}, m_{3}$ are the $U(1), S U(2)$ and $S U(3)$ gaugino masses, $\tan \beta$ the ratio of the Higgs vevs and $\operatorname{sgn}(\mu)$ is the sign of the Higgs mixing parameter appearing in the superpotential, Eq. (3), which is taken to be positive. Using the soft parameters as input at the GUT scale, the renormalization group equations are run down to the electroweak scale using SOFTSUSY 4.1.0 [58,59] which generates the weak scale inputs that enter into the calculation of the observables in this analysis. Also, the SM Higgs boson mass is determined at the two-loop level. The high scale input and the computed Higgs boson masses, consistent with a mass of $125 \pm 2 \mathrm{GeV}$, for several representative benchmark points are presented in Table I.

Since SUSY contributions involve the exchange of scalars (sleptons and sneutrinos), the input of Table I suggests that such a contribution will be suppressed due to the high scalar masses (being in the several TeV range). Hence, we expect the mirror and fourth sequential generations to have a more significant contribution to the observables. The parameters in the vectorlike sector are chosen so as to be consistent with the lepton masses obtained after diagonalization. We present in Table II the results of the observables obtained for three benchmark points, (a), (b) and (c) of Table I. On the rightmost column, the experimental limits on the corresponding

TABLE II. An exhibition of the branching ratios $\mathcal{B}\left(\ell_{i} \rightarrow \ell_{j} \gamma\right)$, electric dipole moments $\left|d_{\alpha}\right|$, anomalous magnetic moments $\Delta a_{\alpha}$ and the Higgs diphoton decay enhancement $R_{\gamma \gamma}$ for three benchmark points (a), (b) and (c) of Table I. For point (a), $\left|f_{3}\right|=2.9,\left|f_{4}\right|=9.3$, $\left|f_{4}^{\prime \prime}\right|=3.5 \times 10^{-3},\left|f_{3}^{\prime \prime}\right|=7.9 \times 10^{-4}, \tilde{M}_{E}=700, \tilde{M}_{\chi}=37300$ for point (b), $\left|f_{3}\right|=3,\left|f_{4}\right|=5,\left|f_{4}^{\prime \prime}\right|=7 \times 10^{-3},\left|f_{3}^{\prime \prime}\right|=7.9 \times 10^{-4}$, $\tilde{M}_{E}=800, \tilde{M}_{\chi}=20500$ and for point (c) $\left|f_{3}\right|=1,\left|f_{4}\right|=25,\left|f_{4}^{\prime \prime}\right|=5 \times 10^{-3},\left|f_{3}^{\prime \prime}\right|=1 \times 10^{-3}, \tilde{M}_{E}=700, \tilde{M}_{\chi}=18100$. The remaining scalar masses and trilinear couplings are taken to be universal at $m_{0}^{V}=5 \times 10^{4}$ and $\left|A_{0}^{V}\right|=8 \times 10^{3}$. Also, common for all points: $\left|f_{3}^{\prime}\right|=1.8 \times 10^{-2}, \quad\left|f_{4}^{\prime}\right|=1.4 \times 10^{-1}, \quad\left|f_{5}\right|=4.5 \times 10^{-8}, \quad\left|f_{5}^{\prime}\right|=3 \times 10^{-8}, \quad\left|f_{5}^{\prime \prime}\right|=1.2 \times 10^{-8}, \quad\left|h_{6}\right|=9.8, \quad\left|h_{7}\right|=2.5$, $\left|h_{8}\right|=498, \quad \alpha_{\mu}=\xi_{1}=\xi_{2}=\alpha_{A_{0}}=\alpha_{A_{\tilde{\nu}}}=0, \quad \chi_{3}=3.1, \quad \chi_{3}^{\prime}=0.2, \quad \chi_{3}^{\prime \prime}=1.1, \quad \chi_{4}=4.7, \quad \chi_{4}^{\prime}=4.0, \chi_{4}^{\prime \prime}=3.9, \chi_{5}=3.6$, $\chi_{5}^{\prime}=3.4, \chi_{5}^{\prime \prime}=1.3, \chi_{6}=3.9, \chi_{7}=1.7, \chi_{8}=6.0, m_{E}=m_{N}=500, m_{G}=400$ and $m_{G}^{\nu}=340$. EDM is in $e$ cm. All masses are in

\begin{tabular}{|c|c|c|c|c|}
\hline Observable & (a) & $\begin{array}{c}\text { Model point } \\
\text { (b) }\end{array}$ & (c) & Upper limits \\
\hline $\mathcal{B}(\mu \rightarrow e \gamma)$ & $3.5 \times 10^{-13}$ & $5.0 \times 10^{-13}$ & $5.6 \times 10^{-13}$ & $5.7 \times 10^{-13}$ \\
\hline $\mathcal{B}(\tau \rightarrow \mu \gamma)$ & $4.1 \times 10^{-8}$ & $3.4 \times 10^{-8}$ & $4.3 \times 10^{-8}$ & $4.4 \times 10^{-8}$ \\
\hline $\mathcal{B}(\tau \rightarrow e \gamma)$ & $3.6 \times 10^{-11}$ & $8.2 \times 10^{-11}$ & $1.2 \times 10^{-10}$ & $3.3 \times 10^{-8}$ \\
\hline$\left|d_{e}\right|$ & $2.4 \times 10^{-29}$ & $4.8 \times 10^{-29}$ & $4.3 \times 10^{-29}$ & $9.3 \times 10^{-29}$ \\
\hline$\left|d_{\mu}\right|$ & $2.1 \times 10^{-26}$ & $2.1 \times 10^{-26}$ & $2.1 \times 10^{-26}$ & $1.9 \times 10^{-19}$ \\
\hline$\left|d_{\tau}\right|$ & $2.5 \times 10^{-23}$ & $1.4 \times 10^{-22}$ & $2.3 \times 10^{-22}$ & $1.1 \times 10^{-17}$ \\
\hline$\left|\Delta a_{\mu}\right|$ & $2.3 \times 10^{-11}$ & $7.1 \times 10^{-12}$ & $1.2 \times 10^{-12}$ & $(28.8 \pm 7.9) \times 10^{-10}$ \\
\hline$\left|\Delta a_{e}\right|$ & $5.4 \times 10^{-16}$ & $1.6 \times 10^{-16}$ & $2.5 \times 10^{-17}$ & $-10.5(8.1) \times 10^{-13}$ \\
\hline$R_{\gamma \gamma}$ & 1.07 & 1.13 & 1.03 & ATLAS/CMS, Eq. (60) \\
\hline
\end{tabular}
$\mathrm{GeV}$ and all phases in rad. 
TABLE III. An exhibition of the branching ratios $\mathcal{B}\left(\ell_{i} \rightarrow \ell_{j} \gamma\right)$, electric dipole moments $\left|d_{\alpha}\right|$, anomalous magnetic moments $\Delta a_{\alpha}$ and the Higgs diphoton decay enhancement $R_{\gamma \gamma}$ for the benchmark point (a) of Table I. The input is $\left|f_{3}\right|=0.3,\left|f_{3}^{\prime}\right|=$ $3.8 \times 10^{2},\left|f_{3}^{\prime \prime}\right|=7.9 \times 10^{-6},\left|f_{4}\right|=9.3 \times 10^{-4},\left|f_{4}^{\prime}\right|=3.2 \times 10^{-1}$, $\left|f_{4}^{\prime \prime}\right|=3.5 \times 10^{-7},\left|f_{5}\right|=4.5 \times 10^{-8},\left|f_{5}^{\prime}\right|=3 \times 10^{-8},\left|f_{5}^{\prime \prime}\right|=$ $1.2 \times 10^{-8},\left|h_{6}\right|=9.8,\left|h_{7}\right|=2.5,\left|h_{8}\right|=498, \alpha_{\mu}=\xi_{1}=\xi_{2}=$ $\alpha_{A_{0}}=\alpha_{A_{\tilde{\nu}}}=0, \chi_{3}=3.1, \chi_{3}^{\prime}=0.2, \chi_{3}^{\prime \prime}=1.1, \chi_{4}=4.7, \chi_{4}^{\prime}=4.0$, $\chi_{4}^{\prime \prime}=3.9, \quad \chi_{5}=3.6, \quad \chi_{5}^{\prime}=3.4, \chi_{5}^{\prime \prime}=1.3, \quad \chi_{6}=3.9, \quad \chi_{7}=1.7$, $\chi_{8}=6.0, \quad m_{E}=m_{N}=500, \quad m_{G}=400 \quad$ and $\quad m_{G}^{\nu}=340$, $\tilde{M}_{E}=700, \tilde{M}_{\chi}=37300$. The remaining scalar masses and trilinear couplings are taken to be universal at $m_{0}^{V}=5 \times 10^{4}$ and $\left|A_{0}^{V}\right|=8 \times 10^{3}$. EDM is in $e \mathrm{~cm}$. All masses are in $\mathrm{GeV}$ and all phases in rad.

\begin{tabular}{lcc}
\hline \hline Observable & Point (a) & Upper limits \\
\hline $\mathcal{B}(\mu \rightarrow e \gamma)$ & $4.0 \times 10^{-14}$ & $5.7 \times 10^{-13}$ \\
$\mathcal{B}(\tau \rightarrow \mu \gamma)$ & $1.3 \times 10^{-8}$ & $4.4 \times 10^{-8}$ \\
$\mathcal{B}(\tau \rightarrow e \gamma)$ & $6.3 \times 10^{-23}$ & $3.3 \times 10^{-8}$ \\
$\left|d_{e}\right|$ & $1.4 \times 10^{-36}$ & $9.3 \times 10^{-29}$ \\
$\left|d_{\mu}\right|$ & $2.2 \times 10^{-22}$ & $1.9 \times 10^{-19}$ \\
$\left|d_{\tau}\right|$ & $1.4 \times 10^{-27}$ & $1.1 \times 10^{-17}$ \\
$\left|\Delta a_{\mu}\right|$ & $2.2 \times 10^{-9}$ & $(28.8 \pm 7.9) \times 10^{-10}$ \\
$\left|\Delta a_{e}\right|$ & $5.4 \times 10^{-16}$ & $-10.5(8.1) \times 10^{-13}$ \\
$R_{\gamma \gamma}$ & 1.07 & ATLAS/CMS, Eq. (60) \\
\hline \hline
\end{tabular}

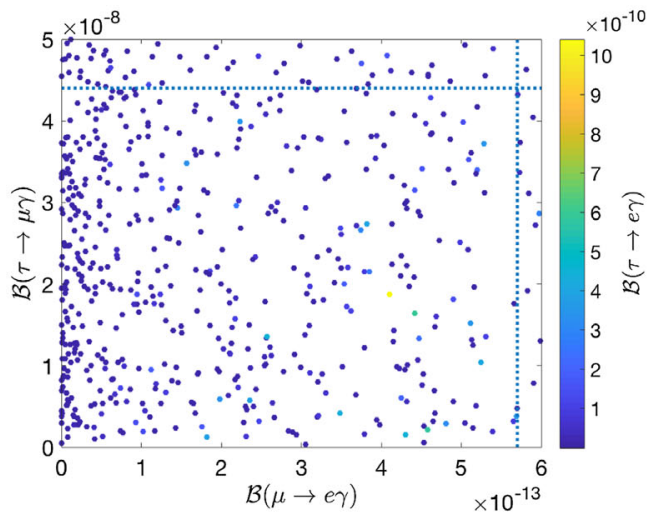

observables are summarized for comparison purpose and the computed values of the observables satisfy these bounds. Thus the branching ratios of $\mu \rightarrow e \gamma$ and $\tau \rightarrow \mu \gamma$ are below but close to their upper limits, especially for points (b) and (c) and could be probed by a small improvement in experiment. The branching ratio of $\tau \rightarrow e \gamma$ appears to be 2 to 3 orders of magnitude smaller than its upper limit. However, one can achieve somewhat higher values by varying the Yukawa masses $m_{E}$ and/or $m_{G}$ as we will see later. It is interesting that for the same parameter set the EDM of the electron is also close to its current limit while the EDMs of the muon and of tau are 5 to 7 orders of magnitude smaller than the upper limits. The electron and muon anomalous magnetic moments are typically small and the contribution is not significant to explain the $\sim 3 \sigma$ deviation if indeed it holds up in improved experiment. As for the diphoton rate enhancement there are discernible corrections to the branching ratio but consistent with the current limits from ATLAS and CMS, Eq. (60). Here we note that it was shown in previous works (see, e.g., [46]) that a muon $g-2$ close to the experimental limit can be obtained via leptonic vectorlike exchange. To see if this is possible with the current constraints we take point (a) from Table II and modify the input parameters. The results are listed in Table III where a muon $g-2$ of $\mathcal{O}\left(10^{-9}\right)$ and within the observed $3 \sigma$ deviation is obtained. The rest of the
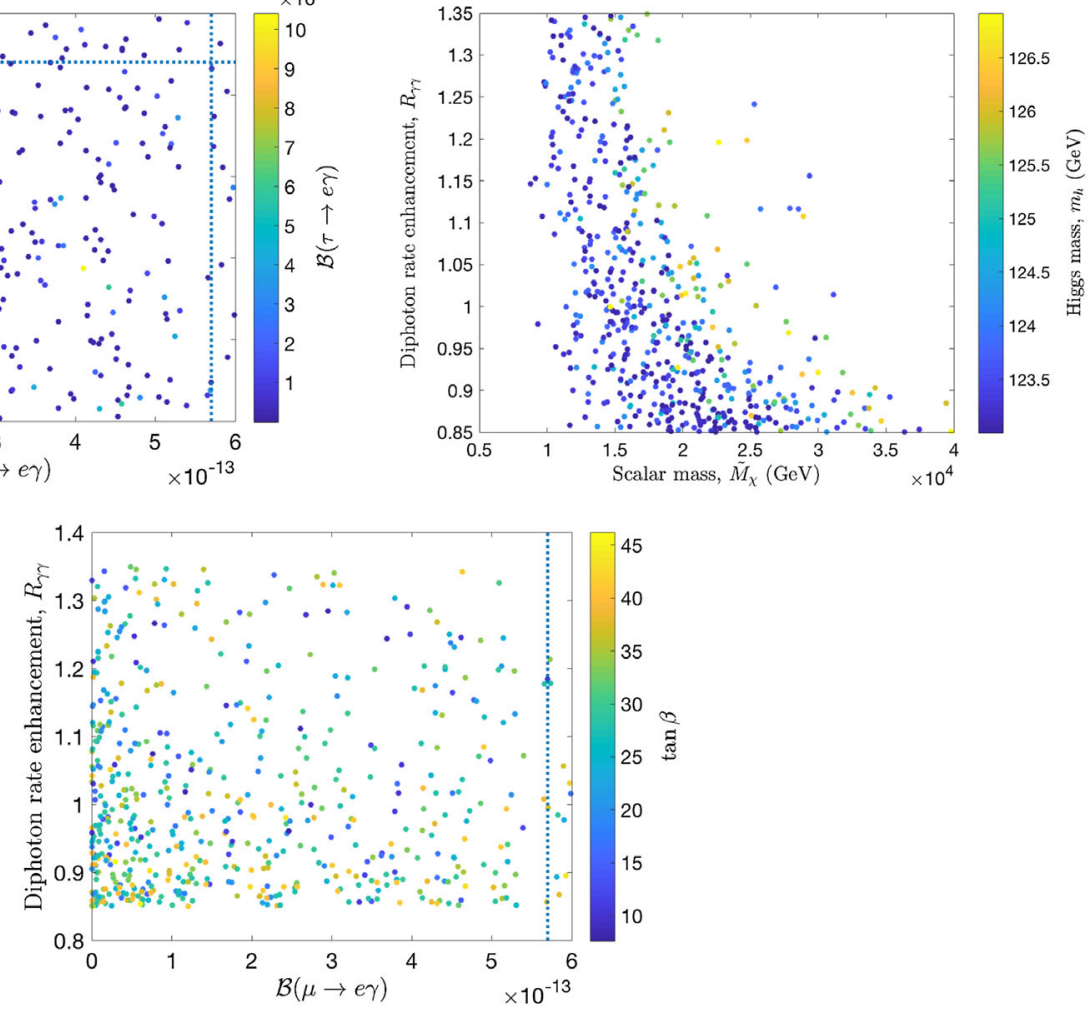

FIG. 5. Scatter plots for different observables where the scan is performed over all couplings and their phases for a set of SUGRA benchmark points satisfying the Higgs mass. The upper left panel shows all branching ratios, the upper right panel displays the diphoton enhancement factor, $R_{\gamma \gamma}$ for different values of the scalar mass, $\tilde{M}_{\chi}$, and the Higgs mass. In the bottom panel, a display of $R_{\gamma \gamma}$ and $\mathcal{B}(\mu \rightarrow e \gamma)$ for different $\tan \beta$. Dashed vertical and horizontal lines correspond to experimental upper limits on the corresponding observables. 

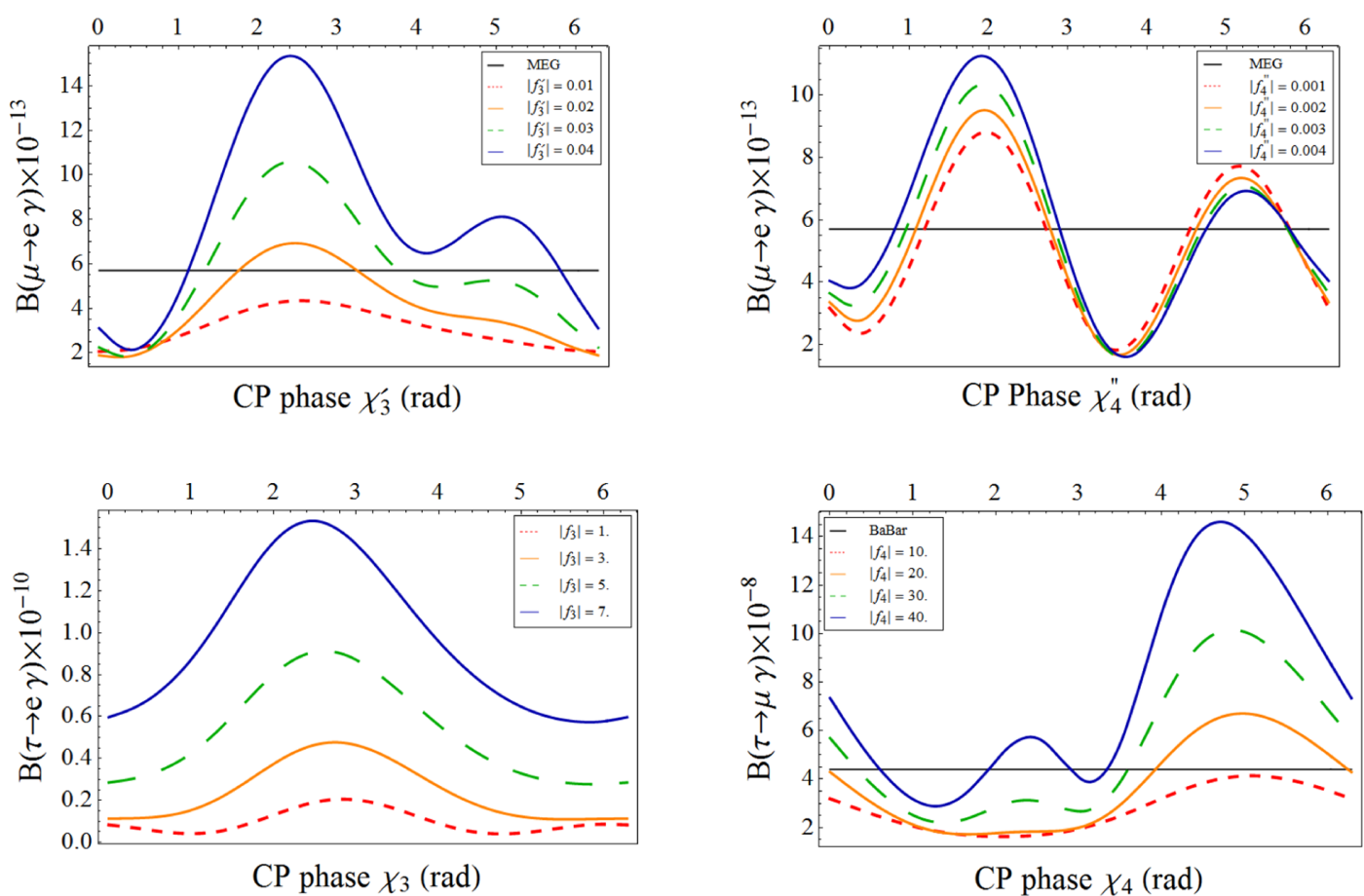

CP phase $\chi_{4}(\mathrm{rad})$

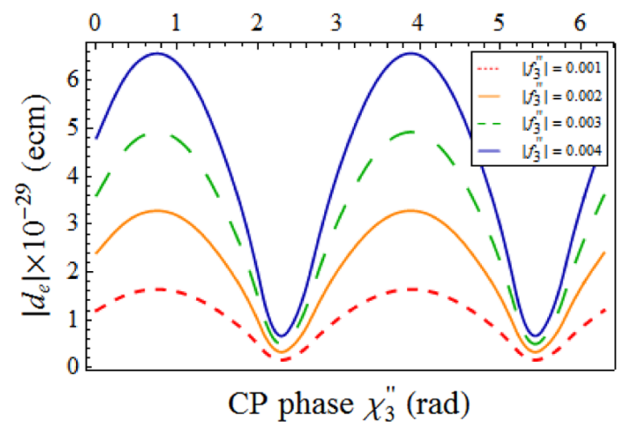

FIG. 6. An exhibition of the branching ratios and electron EDM versus the $C P$ phases for point (a) of Table I. The upper panels show $\mathcal{B}(\mu \rightarrow e \gamma)$ as a function of the $C P$ phases $\chi_{3}^{\prime}$ and $\chi_{4}^{\prime \prime}$ for different values of $\left|f_{3}^{\prime}\right|$ and $\left|f_{4}^{\prime \prime}\right|$, respectively. The middle panels show $\mathcal{B}(\tau \rightarrow e \gamma)$ and $\mathcal{B}(\tau \rightarrow \mu \gamma)$ as a function of the $C P$ phases $\chi_{3}$ and $\chi_{4}$ for different values of $\left|f_{3}\right|$ and $\left|f_{4}\right|$, respectively. The bottom panel displays the electron EDM versus $\chi_{3}^{\prime \prime}$ for different values of $\left|f_{3}^{\prime \prime}\right|$. All other parameters are the same as for point (a) in Table II.

observables are still in check but one of the branching ratios, namely, $\tau \rightarrow e \gamma$, has become very small. Also, we have obtained a 4 orders of magnitude increase in the muon EDM.

While the results presented here are for an explicit sample set, we have analyzed the parameter space of the model much more widely in the ranges displayed in Eq. (74),

$$
\begin{aligned}
& \left|f_{3}\right| \in[1,10], \quad\left|f_{3}^{\prime}\right| \in\left[1 \times 10^{-2}, 10\right], \\
& \left|f_{3}^{\prime \prime}\right| \in\left[1 \times 10^{-6}, 1 \times 10^{-4}\right], \\
& \left|f_{4}\right| \in[1,20], \quad\left|f_{4}^{\prime}\right| \in\left[1 \times 10^{-2}, 10\right], \\
& \left|f_{3}^{\prime \prime}\right| \in\left[1 \times 10^{-5}, 1 \times 10^{-2}\right], \\
& \left|h_{6}\right| \in[1,20], \quad\left|h_{7}\right| \in\left[1 \times 10^{-3}, 10\right], \quad\left|h_{8}\right| \in[5,600], \\
& \tilde{M}_{E} \in[600,800], \quad \tilde{M}_{\chi} \in[3,5] \times 10^{4}, \quad \chi_{i} \in[0,2 \pi],
\end{aligned}
$$

where the vectorlike Yukawa masses are fixed so that $m_{E}=m_{N}=500 \mathrm{GeV}, m_{G}=400 \mathrm{GeV}$ and $m_{G}^{\nu}=340 \mathrm{GeV}$. The couplings $\left|f_{5}\right|,\left|f_{5}^{\prime}\right|$ and $\left|f_{5}^{\prime \prime}\right|$ are kept small, i.e., $\mathcal{O}\left(10^{-8}\right)$. The scan results in 17 million points but is greatly reduced when the constraints on the nine observables are applied. The results are displayed as scatter plots in Fig. 5.

Thus, in the upper left panel of Fig. 5 we display a scatter plot in the three observables, $\mathcal{B}(\mu \rightarrow$ er $)$, $\mathcal{B}(\tau \rightarrow \mu \gamma)$ and $\mathcal{B}(\tau \rightarrow e \gamma)$. The dashed vertical and horizontal lines are the upper limits on $\mathcal{B}(\mu \rightarrow e \gamma)$ and $\mathcal{B}(\tau \rightarrow \mu \gamma)$, respectively. One can see that there are plenty of points below but close to the upper limits while satisfying all the other observables. The upper right and bottom panels show scatter plots in $R_{\gamma \gamma}$ versus the scalar mass from the vectorlike sector, $\tilde{M}_{\chi}$ in one and $\mathcal{B}(\mu \rightarrow e \gamma)$ in the other. The Higgs boson mass and $\tan \beta$ are also shown in the 

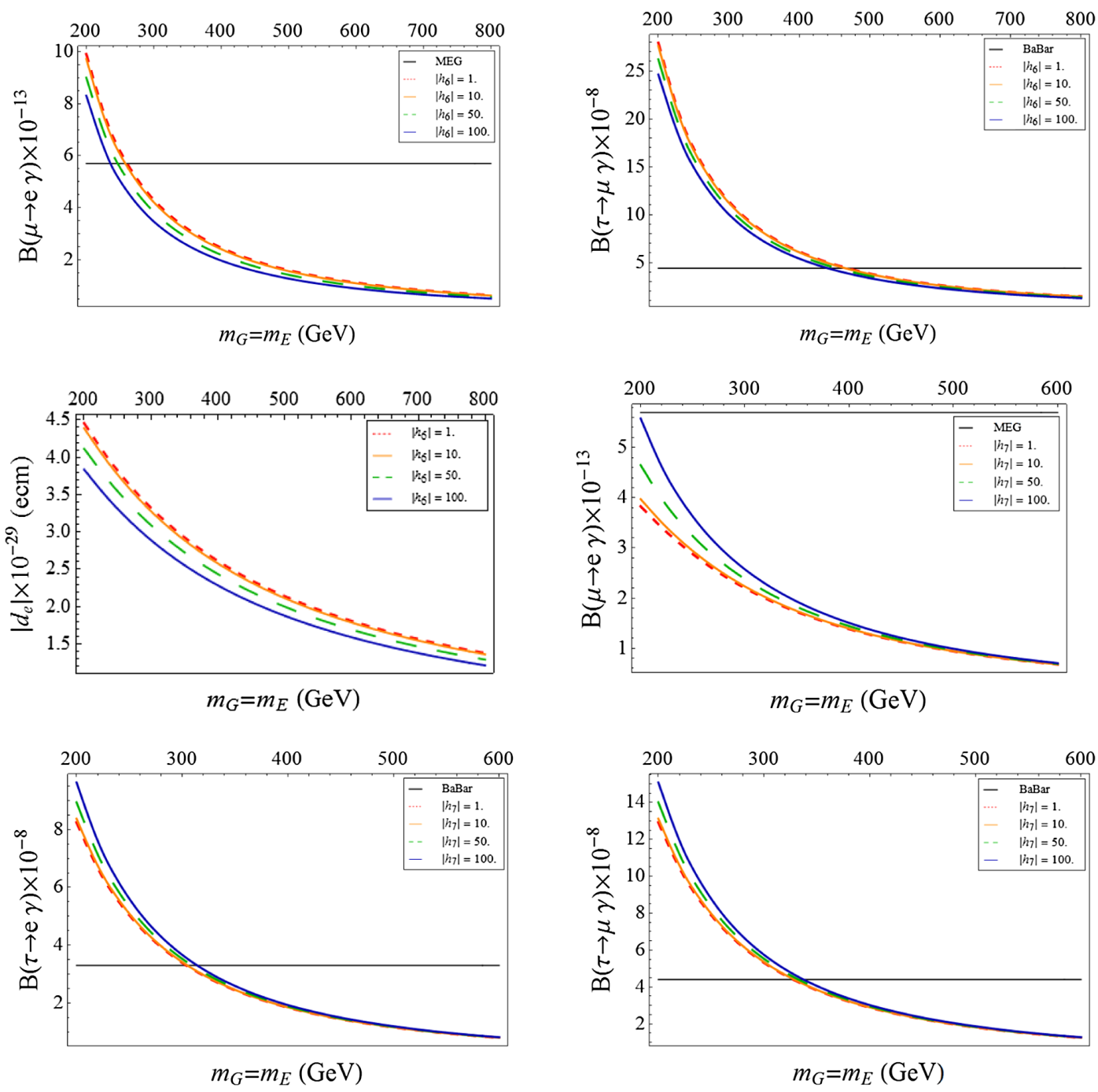

FIG. 7. An exhibition of the $\mu \rightarrow e \gamma$ and $\tau \rightarrow \mu \gamma$ branching ratios (top panel) and the electron EDM (middle-left panel) as a function of $m_{E}\left(=m_{G}\right)$ for different values of $\left|h_{6}\right|$ for benchmark point (c) while plots of all branching ratios (middle-right and bottom panels) are drawn for different $\left|h_{7}\right|$ for point (e) of Table I. For point (c), all other parameters are the same as in Table II. As for point (e), the other scalar masses are $m_{0}^{V}=5 \times 10^{4}$ except for $\tilde{M}_{E}=700$ and $\tilde{M}_{\chi}=18100$ and the rest of the trilinear couplings are $\left|A_{0}^{V}\right|=8 \times 10^{3}$. Also, $\left|f_{3}\right|=7,\left|f_{3}^{\prime}\right|=2 \times 10^{-3},\left|f_{3}^{\prime \prime}\right|=2 \times 10^{-5},\left|f_{4}\right|=4 \times 10^{-1},\left|f_{4}^{\prime}\right|=5 \times 10^{-2},\left|f_{4}^{\prime \prime}\right|=4 \times 10^{-2},\left|f_{5}\right|=4.5 \times 10^{-10},\left|f_{5}^{\prime}\right|=3 \times 10^{-10}$, $\left|f_{5}^{\prime \prime}\right|=1.2 \times 10^{-10},\left|h_{6}\right|=9.8,\left|h_{8}\right|=4.98 \times 10^{2}, M_{N}=500, m_{G}^{\nu}=340, \chi_{3}=3.1, \chi_{3}^{\prime}=0.2, \chi_{3}^{\prime \prime}=1.1, \chi_{4}=-1.58, \chi_{4}^{\prime}=-2.3$, $\chi_{4}^{\prime \prime}=-2.35, \chi_{5}=-2.7, \chi_{5}^{\prime}=-2.9, \chi_{5}^{\prime \prime}=1.3, \chi_{6}=-2.4, \chi_{7}=1.7$ and $\chi_{8}=-0.3$. All masses are in GeV and phases in rad.

$z$-direction. One can see that values of $R_{\gamma \gamma}$ within the experimental limits are more favorable for lower $\tilde{M}_{\chi}$ values. The reason for this is the following: For vectorlike masses much smaller than their Yukawa counterparts, i.e., $\left|h_{6}\right|\left|h_{7}\right| \ll \frac{1}{2} f_{2}^{\prime} y_{5} v_{1} v_{2}$, the fermionic contribution, $r_{f}$ in Eq. (66), to the diphoton rate enhancement is negative and large $(\sim-0.4$ for the parameter space under consideration). To get values of $R_{\gamma \gamma}$ consistent with experiment, a positive and large contribution must come from the bosonic part, $r_{b}$, Eq. (70). It is shown that smaller values of $\tilde{M}_{E}$ and $\tilde{M}_{\chi}$, in the range given by Eq. (74), can achieve this purpose without affecting other observables. Having this range of values means lighter vectorlike superpartners and the loop contributions become less suppressed. Since the SUSY loops are suppressed, the vectorlike sector is the largest contributor to the various observables considered here.

We discuss now in further detail the sensitivity of some of the observables on the various input parameters. Thus in Fig. 6 we display the variation of $\mathcal{B}(\mu \rightarrow e \gamma)$, $\mathcal{B}(\tau \rightarrow e \gamma), \mathcal{B}(\tau \rightarrow \mu \gamma)$ and the electron EDM, $\left|d_{e}\right|$ as a function of the $C P$ phases from the vectorlike sector. It is clear that all those observables exhibit a sensitive dependence on the $C P$ phases where the branching ratios oscillate above and below their upper limits. Also, the electron EDM shows large variations very close to the experimental upper limit. The different curves in each plot correspond to different choices of the couplings 

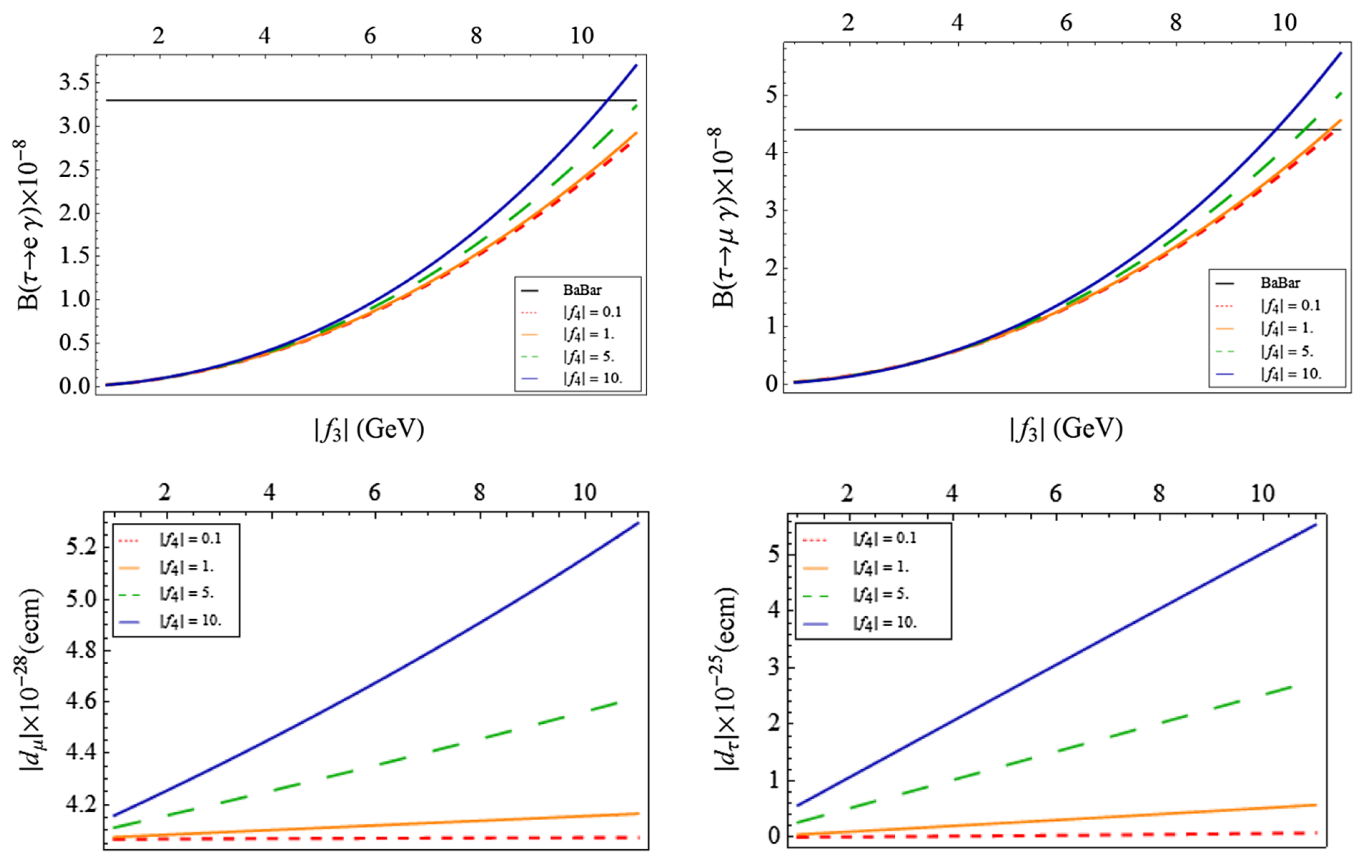

$\left|f_{3}\right|(\mathrm{GeV})$

$\left|f_{3}\right|(\mathrm{GeV})$

FIG. 8. An exhibition of the $\tau \rightarrow e \gamma$ and $\tau \rightarrow \mu \gamma$ branching ratios and the muon and tau EDMs as a function of $\left|f_{3}\right|$ for different values of $\left|f_{4}\right|$ for point (d) of Table I. The other scalar masses are $m_{0}^{V}=5 \times 10^{4}$ except for $\tilde{M}_{E}=700$ and $\tilde{M}_{\chi}=18100$ and the rest of the trilinear couplings are $\left|A_{0}^{V}\right|=8 \times 10^{3}$. Also, $\left|f_{3}^{\prime}\right|=2 \times 10^{-3},\left|f_{3}^{\prime \prime}\right|=2 \times 10^{-5},\left|f_{4}^{\prime}\right|=5 \times 10^{-2},\left|f_{4}^{\prime \prime}\right|=4 \times 10^{-2},\left|f_{5}\right|=4.5 \times 10^{-10}$, $\left|f_{5}^{\prime}\right|=3 \times 10^{-10},\left|f_{5}^{\prime \prime}\right|=1.2 \times 10^{-10},\left|h_{6}\right|=9.8,\left|h_{7}\right|=2.5,\left|h_{8}\right|=4.98 \times 10^{2}, M_{N}=m_{E}=500, m_{G}=400, m_{G}^{\nu}=340, \chi_{3}=3.1$, $\chi_{3}^{\prime}=0.2, \chi_{3}^{\prime \prime}=1.1, \chi_{4}=-1.58, \chi_{4}^{\prime}=-2.3, \chi_{4}^{\prime \prime}=-2.35, \chi_{5}=-2.7, \chi_{5}^{\prime}=-2.9, \chi_{5}^{\prime \prime}=1.3, \chi_{6}=-2.4, \chi_{7}=1.7$ and $\chi_{8}=-0.3$. All masses are in $\mathrm{GeV}$ and phases in rad.

$\left|f_{3}\right|,\left|f_{3}^{\prime}\right|,\left|f_{3}^{\prime \prime}\right|,\left|f_{4}\right|$ and $\left|f_{4}^{\prime \prime}\right|$ where larger values of the observables are obtained for larger couplings. Note that those couplings cannot take arbitrarily large values since this will spoil the lepton masses.

In Fig. 7 we show the dependence of the branching ratios of $\mu \rightarrow e \gamma, \tau \rightarrow \mu \gamma, \tau \rightarrow e \gamma$ and the electron EDM on the vectorlike Yukawa masses for points (c) and (e) of Table I. The observables show a decaying trend for larger values of the masses which is due to larger suppression of loop effects due to the exchange of heavier particles. For point (c), the branching ratio of $\mu \rightarrow e \gamma$ drops below its upper limit for a mass $\sim 250 \mathrm{GeV}$ while $\tau \rightarrow \mu \gamma$ does that for a heavier mass, $\sim 450 \mathrm{GeV}$ (top panel). The different curves in each plot correspond to different choices of the vectorlike mass $\left|h_{6}\right|$ where, as one would expect, the contribution from the vectorlike sector is larger for smaller values of $\left|h_{6}\right|$. The interesting aspect of point (e) is in the variation of the branching ratios (middle-right and bottom panels) against $m_{E}$. As Fig. 7 shows, one can simultaneously get all three branching ratios just below their upper limits by choosing particular values of $m_{E}=m_{G}$ and $\left|h_{7}\right|$. Thus, $\mathcal{B}(\tau \rightarrow$ $e \gamma)$ and $\mathcal{B}(\tau \rightarrow \mu \gamma)$ plunge right below their upper limits at around $350 \mathrm{GeV}$ while $\mathcal{B}(\mu \rightarrow e \gamma)$ is already below the upper limit for even smaller $m_{E}=m_{G}$. This shows how the interplay of those parameters lead to all constraints to fall in place. While it was difficult to achieve larger $\mathcal{B}(\tau \rightarrow e \gamma)$ values (in Table II), it was easier to do so for point (e).

The coupling $f_{3}$ mixes the vectorlike generation with the first leptonic generation of Eq. (10). Thus we expect this coupling to have the largest impact on observables pertaining to the $\tau$ lepton. To check this, we exhibit the variation of the radiative tau decay branching ratios, the muon EDM and tau EDM against $\left|f_{3}\right|$ in Fig. 8. The plots are drawn for different values of $\left|f_{4}\right|$. As one can clearly see, the branching ratios of tau and the tau EDM are impacted the most where the former observables may shoot above their upper limits for higher values of $\left|f_{3}\right|$, while the variation of the muon EDM is rather mild. Larger values of $\left|f_{4}\right|$, which couples the vectorlike and first generation singlet fields, produce larger values of the considered observables as one would expect as well.

In the above we discussed the lepton flavor changing process $\mu \rightarrow e \gamma$ but did not discuss the flavor changing processes $\mu \rightarrow e$ conversion and $\mu \rightarrow 3 e$. A proper treatment of these processes at the same level of care as done for the other processes treated here is outside the scope of this work. Thus, e.g., for the $\mu \rightarrow e$ conversion one needs computation of a set of box and penguin diagrams which would again involve our $10 \times 10$ scalar mass matrices in 
the loops. In addition $\mu \rightarrow e$ conversion has much more model dependence because of nuclear physics effects. Here we give approximate results for them valid in certain limits which, however, do indicate the expected size of the branching ratios for these processes for the parameter sets in our case given in Table II. Thus in the dipole dominance approximation, one has [60]

$$
\frac{\mathcal{B}(\mu \rightarrow 3 e)}{\mathcal{B}(\mu \rightarrow e \gamma)} \simeq \frac{\alpha_{e m}}{3 \pi}\left(\log \frac{m_{\mu}^{2}}{m_{e}^{2}}-\frac{11}{4}\right) .
$$

The right-hand side of Eq. (75) evaluates to $\sim 6 \times 10^{-3}$. Using this ratio $\mathcal{B}(\mu \rightarrow 3 e) \sim 3.4 \times 10^{-15}$ for column 3 in Table II. This is to be compared with the current experimental limit [61]

$$
\mathcal{B}(\mu \rightarrow 3 e)<1.0 \times 10^{-12} \text { at } 90 \% \text { C.L. }
$$

In future experiments [62] this limit may reach $\mathcal{B}(\mu \rightarrow 3 e) \leq 10^{-16}$. For the $\mu \rightarrow e$ conversion process the analysis of [63] in the limit $m_{\chi^{ \pm}}^{2} / m_{\tilde{e}}^{2} \sim 1$ gives $\mathcal{B}(\mu \rightarrow e)_{N} / \mathcal{B}(\mu \rightarrow e \gamma) \sim \alpha_{e m} / 3$ for aluminum and $\mathcal{B}(\mu \rightarrow e)_{N} / \mathcal{B}(\mu \rightarrow e \gamma) \sim \alpha_{e m} / 2$ for gold. Numerically, for aluminum this gives $\mathcal{B}(\mu \rightarrow e)_{\mathrm{Al}} \sim 1.3 \times 10^{-15}$ and for gold it gives $\mathcal{B}(\mu \rightarrow e)_{\mathrm{G}} \sim 1.95 \times 10^{-15}$ for the third column in Table II. The current experimental limit for $\mu \rightarrow e$ conversion for gold is [64]

$$
\mathcal{B}(\mu \rightarrow e)_{\mathrm{Au}}<7 \times 10^{-13} \quad 90 \% \text { C.L. }
$$

In the future one expects that experiments using $\mathrm{Al}$ nuclei will reach a sensitivity in the range $[31,65] \mathcal{B}(\mu \rightarrow e)_{\mathrm{Al}}<$ $10^{-16}-10^{-18}$.

\section{CONCLUSION}

In a large class of models such as based on grand unification, on strings and branes, one has vectorlike states some of which could be light and lie in the low energy region accessible to experiment. Their presence can affect low energy phenomena through loop corrections. In supersymmetric theories the vectorlike generations will have particles and their mirrors as well as sparticles and their mirrors. This means that in a model with three generations there will be two more particles that can appear in the mixing matrix, making the fermionic mixing matrix a $5 \times 5$ mixing matrix. In the slepton sector, one will have in general a $10 \times 10$ mixing matrix. The analysis is done including the $C P$ violating phases in the mixings of the vectorlike generation. In his work we have carried out a correlated study of the effects of the vectorlike generation on several observables. These include $\mu \rightarrow e \gamma, \tau \rightarrow \mu \gamma$, $\tau \rightarrow e \gamma$, muon and electron magnetic moments, $g_{\mu}-2$ and $g_{e}-2$, and EDMs of the charged leptons $d_{e}, d_{\mu}, d_{\tau}$. We also examine the effect of the vectorlike generation on $h \rightarrow \gamma \gamma$. The analysis is done under the constraints of the Higgs boson mass at $125 \mathrm{GeV}$. Several interesting correlations are observed which are discussed in the numerical section. In the coming years improvement in experiment on several fronts will occur and the predictions of the vectorlike generations can be checked or the model further constrained.

\section{ACKNOWLEDGMENTS}

This research was supported in part by NSF Grant No. PHY-1620526.

\section{APPENDIX A: THE EXTENDED MSSM WITH A VECTORLIKE LEPTONIC GENERATION}

The mass-squared matrices of the supersymmetric scalar sectors (sleptons, sneutrinos and their mirrors) arise from the $F$ and $D$ terms of the potential and from the soft SUSY breaking terms such that $\mathcal{L}_{\text {soft }}$ takes the form

$$
\begin{aligned}
-\mathcal{L}_{\text {soft }}= & \tilde{M}_{\tau L}^{2} \tilde{\psi}_{\tau L}^{i *} \tilde{\psi}_{\tau L}^{i}+\tilde{M}_{\chi}^{2} \tilde{\chi}^{c i *} \tilde{\chi}^{c i}+\tilde{M}_{\mu L}^{2} \tilde{\psi}_{\mu L}^{i *} \tilde{\psi}_{\mu L}^{i}+\tilde{M}_{e L}^{2} \tilde{\psi}_{e L}^{i *} \tilde{\psi}_{e L}^{i}+\tilde{M}_{\nu_{\tau}}^{2} \tilde{\nu}_{\tau L}^{c *} \tilde{\nu}_{\tau L}^{c}+\tilde{M}_{\nu_{\mu}}^{2} \tilde{\nu}_{\mu L}^{c *} \tilde{\nu}_{\mu L}^{c}+\tilde{M}_{4 L}^{2} \tilde{\psi}_{4 L}^{i *} \tilde{\psi}_{4 L}^{i}+\tilde{M}_{\nu_{4}}^{2} \tilde{\nu}_{4 L}^{c *} \tilde{\nu}_{4 L}^{c} \\
& +\tilde{M}_{\nu_{e}}^{2} \tilde{\nu}_{e L}^{c *} \tilde{\nu}_{e L}^{c}+\tilde{M}_{\tau}^{2} \tilde{\tau}_{L}^{c *} \tilde{\tau}_{L}^{c}+\tilde{M}_{\mu}^{2} \tilde{\mu}_{L}^{c *} \tilde{\mu}_{L}^{c}+\tilde{M}_{e}^{2} \tilde{e}_{L}^{c *} \tilde{e}_{L}^{c}+\tilde{M}_{E}^{2} \tilde{E}_{L}^{*} \tilde{E}_{L}+\tilde{M}_{N}^{2} \tilde{N}_{L}^{*} \tilde{N}_{L}+\tilde{M}_{4}^{2} \tilde{\ell}_{4 L}^{c *} \tilde{\ell}_{4 L}^{c} \\
& +\epsilon_{i j}\left\{f_{1} A_{\tau} H_{1}^{i} \tilde{\psi}_{\tau L}^{j} \tilde{\tau}_{L}^{c}-f_{1}^{\prime} A_{\nu_{\tau}} H_{2}^{i} \tilde{\psi}_{\tau L}^{j} \tilde{\nu}_{\tau L}^{c}+h_{1} A_{\mu} H_{1}^{i} \tilde{\psi}_{\mu L}^{j} \tilde{\mu}_{L}^{c}-h_{1}^{\prime} A_{\nu_{\mu}} H_{2}^{i} \tilde{\psi}_{\mu L}^{j} \tilde{\nu}_{\mu L}^{c}+h_{2} A_{e} H_{1}^{i} \tilde{\psi}_{e L}^{j} \tilde{e}_{L}^{c}-h_{2}^{\prime} A_{\nu_{e}} H_{2}^{i} \tilde{\psi}_{e L}^{j} \tilde{\nu}_{e L}^{c}\right. \\
& \left.+f_{2} A_{N} H_{1}^{i} \tilde{\chi}^{c j} \tilde{N}_{L}-f_{2}^{\prime} A_{E} H_{2}^{i} \tilde{\chi}^{c j} \tilde{E}_{L}+y_{5} A_{4 \ell} H_{1}^{i} \tilde{\psi}_{4 L}^{j} \tilde{\ell}_{4 L}^{c}-y_{5}^{\prime} A_{4 L} H_{2}^{i} \tilde{\psi}_{4 L}^{j} \tilde{\nu}_{4 L}^{c}+\text { H.c. }\right\} .
\end{aligned}
$$

We define the slepton mass-squared matrix $M_{\tilde{\tau}}^{2}$ in the basis

$$
\left(\tilde{\tau}_{L}, \tilde{E}_{L}, \tilde{\tau}_{R}, \tilde{E}_{R}, \tilde{\mu}_{L}, \tilde{\mu}_{R}, \tilde{e}_{L}, \tilde{e}_{R}, \tilde{\ell}_{4 L}, \tilde{\ell}_{4 R}\right)
$$

and label the matrix elements as $\left(M_{\tilde{\tau}}^{2}\right)_{i j}=M_{i j}^{2}$ where these elements are given by 


$$
\begin{aligned}
& M_{11}^{2}=\tilde{M}_{\tau L}^{2}+\frac{v_{1}^{2}\left|f_{1}\right|^{2}}{2}+\left|f_{3}\right|^{2}-m_{Z}^{2} \cos 2 \beta\left(\frac{1}{2}-\sin ^{2} \theta_{W}\right), \\
& M_{22}^{2}=\tilde{M}_{E}^{2}+\frac{v_{2}^{2}\left|f_{2}^{\prime}\right|^{2}}{2}+\left|f_{4}\right|^{2}+\left|f_{4}^{\prime}\right|^{2}+\left|f_{4}^{\prime \prime}\right|^{2}+\left|h_{7}\right|^{2}+m_{Z}^{2} \cos 2 \beta \sin ^{2} \theta_{W} \\
& M_{33}^{2}=\tilde{M}_{\tau}^{2}+\frac{v_{1}^{2}\left|f_{1}\right|^{2}}{2}+\left|f_{4}\right|^{2}-m_{Z}^{2} \cos 2 \beta \sin ^{2} \theta_{W} \\
& M_{44}^{2}=\tilde{M}_{\chi}^{2}+\frac{v_{2}^{2}\left|f_{2}^{\prime}\right|^{2}}{2}+\left|f_{3}\right|^{2}+\left|f_{3}^{\prime}\right|^{2}+\left|f_{3}^{\prime \prime}\right|^{2}+\left|h_{6}\right|^{2}+m_{Z}^{2} \cos 2 \beta\left(\frac{1}{2}-\sin ^{2} \theta_{W}\right), \\
& M_{55}^{2}=\tilde{M}_{\mu L}^{2}+\frac{v_{1}^{2}\left|h_{1}\right|^{2}}{2}+\left|f_{3}^{\prime}\right|^{2}-m_{Z}^{2} \cos 2 \beta\left(\frac{1}{2}-\sin ^{2} \theta_{W}\right) \\
& M_{66}^{2}=\tilde{M}_{\mu}^{2}+\frac{v_{1}^{2}\left|h_{1}\right|^{2}}{2}+\left|f_{4}^{\prime}\right|^{2}-m_{Z}^{2} \cos 2 \beta \sin ^{2} \theta_{W} \\
& M_{77}^{2}=\tilde{M}_{e L}^{2}+\frac{v_{1}^{2}\left|h_{2}\right|^{2}}{2}+\left|f_{3}^{\prime \prime}\right|^{2}-m_{Z}^{2} \cos 2 \beta\left(\frac{1}{2}-\sin ^{2} \theta_{W}\right), \\
& M_{88}^{2}=\tilde{M}_{e}^{2}+\frac{v_{1}^{2}\left|h_{2}\right|^{2}}{2}+\left|f_{4}^{\prime \prime}\right|^{2}-m_{Z}^{2} \cos 2 \beta \sin ^{2} \theta_{W} \\
& M_{99}^{2}=\tilde{M}_{4 L}^{2}+\frac{v_{1}^{2}\left|y_{5}\right|^{2}}{2}+\left|h_{6}\right|^{2}-m_{Z}^{2} \cos 2 \beta\left(\frac{1}{2}-\sin ^{2} \theta_{W}\right) \text {, } \\
& M_{1010}^{2}=\tilde{M}_{4}^{2}+\frac{v_{1}^{2}\left|y_{5}\right|^{2}}{2}+\left|h_{7}\right|^{2}-m_{Z}^{2} \cos 2 \beta \sin ^{2} \theta_{W}, \quad M_{12}^{2}=M_{21}^{2 *}=\frac{v_{2} f_{2}^{\prime} f_{3}^{*}}{\sqrt{2}}+\frac{v_{1} f_{4} f_{1}^{*}}{\sqrt{2}}, \\
& M_{13}^{2}=M_{31}^{2 *}=\frac{f_{1}^{*}}{\sqrt{2}}\left(v_{1} A_{\tau}^{*}-\mu v_{2}\right), \quad M_{14}^{2}=M_{41}^{2 *}=0, \quad M_{15}^{2}=M_{51}^{2 *}=f_{3}^{\prime} f_{3}^{*}, \quad M_{16}^{2}=M_{61}^{2 *}=0, \\
& M_{17}^{2}=M_{71}^{2 *}=f_{3}^{\prime \prime} f_{3}^{*}, \quad M_{18}^{2}=M_{81}^{2 *}=0, \quad M_{23}^{2}=M_{32}^{2 *}=0, \\
& M_{24}^{2}=M_{42}^{2 *}=\frac{f_{2}^{\prime *}}{\sqrt{2}}\left(v_{2} A_{E}^{*}-\mu v_{1}\right), \quad M_{25}^{2}=M_{52}^{2 *}=\frac{v_{2} f_{3}^{\prime} f_{2}^{\prime *}}{\sqrt{2}}+\frac{v_{1} h_{1} f_{4}^{*}}{\sqrt{2}}, \\
& M_{26}^{2}=M_{62}^{2 *}=0, \quad M_{27}^{2}=M_{72}^{2 *}=\frac{v_{2} f_{3}^{\prime \prime} f_{2}^{\prime *}}{\sqrt{2}}+\frac{v_{1} h_{1} f_{4}^{\prime *}}{\sqrt{2}}, \quad M_{28}^{2}=M_{82}^{2 *}=0, \\
& M_{34}^{2}=M_{43}^{2 *}=\frac{v_{2} f_{4} f_{2}^{*}}{\sqrt{2}}+\frac{v_{1} f_{1} f_{3}^{*}}{\sqrt{2}}, \quad M_{35}^{2}=M_{53}^{2 *}=0, \quad M_{36}^{2}=M_{63}^{2 *}=f_{4} f_{4}^{\prime *}, \\
& M_{37}^{2}=M_{73}^{2 *}=0, \quad M_{38}^{2}=M_{83}^{2 *}=f_{4} f_{4}^{\prime \prime *}, \quad M_{45}^{2}=M_{54}^{2 *}=0, \quad M_{46}^{2}=M_{64}^{2 *}=\frac{v_{2} f_{2}^{\prime} f_{4}^{\prime *}}{\sqrt{2}}+\frac{v_{1} f_{3}^{\prime} h_{1}^{*}}{\sqrt{2}}, \\
& M_{47}^{2}=M_{74}^{2 *}=0, \quad M_{48}^{2}=M_{84}^{2 *}=\frac{v_{2} f_{2}^{\prime} f_{4}^{\prime \prime *}}{\sqrt{2}}+\frac{v_{1} f_{3}^{\prime \prime} h_{2}^{*}}{\sqrt{2}}, \\
& M_{56}^{2}=M_{65}^{2 *}=\frac{h_{1}^{*}}{\sqrt{2}}\left(v_{1} A_{\mu}^{*}-\mu v_{2}\right), \quad M_{57}^{2}=M_{75}^{2 *}=f_{3}^{\prime \prime} f_{3}^{\prime *}, \quad M_{58}^{2}=M_{85}^{2 *}=0, \quad M_{67}^{2}=M_{76}^{2 *}=0, \\
& M_{68}^{2}=M_{86}^{2 *}=f_{4}^{\prime} f_{4}^{\prime \prime *}, \quad M_{78}^{2}=M_{87}^{2 *}=\frac{h_{2}^{*}}{\sqrt{2}}\left(v_{1} A_{e}^{*}-\mu v_{2}\right) \quad M_{19}^{2}=M_{91}^{2 *}=f_{3}^{*} h_{6}, \quad M_{110}^{2}=M_{101}^{2 *}=0, \\
& M_{29}^{2}=M_{92}^{2 *}=\frac{v_{1} y_{5} h_{7}^{*}}{\sqrt{2}}+\frac{v_{2} h_{6} f_{2}^{\prime *}}{\sqrt{2}}, \quad M_{210}^{2}=M_{102}^{2 *}=0, \quad M_{39}^{2}=M_{93}^{2 *}=0, \quad M_{310}^{2}=M_{103}^{2 *}=f_{4} h_{7}^{*}, \\
& M_{49}^{2}=M_{94}^{2 *}=0, \quad M_{410}^{2}=M_{104}^{2 *}=\frac{v_{2} f_{2}^{\prime} h_{7}^{*}}{\sqrt{2}}+\frac{v_{1} h_{6} y_{5}^{*}}{\sqrt{2}}, \quad M_{59}^{2}=M_{95}^{2 *}=f_{3}^{* *} h_{6}, \quad M_{510}^{2}=M_{105}^{2 *}=0, \\
& M_{69}^{2}=M_{96}^{2 *}=0, \quad M_{610}^{2}=M_{106}^{2 *}=f_{4}^{\prime} h_{7}^{*}, \quad M_{79}^{2}=M_{97}^{2 *}=f_{3}^{\prime \prime *} h_{6}, \quad M_{710}^{2}=M_{107}^{2 *}=0, \\
& M_{89}^{2}=M_{98}^{2 *}=0, \quad M_{810}^{2}=M_{108}^{2 *}=f_{5}^{\prime \prime} h_{7}^{*}, \quad M_{910}^{2}=M_{109}^{2 *}=\frac{y_{5}^{*}}{\sqrt{2}}\left(v_{1} A_{4 \ell}^{*}-\mu v_{2}\right) \text {. }
\end{aligned}
$$


We assume that the masses that enter the mass-squared matrix for the scalars are all of electroweak size. This mass-squared matrix is Hermitian and can be diagonalized with a unitary transformation:

$$
\tilde{D}^{\tau \dagger} M_{\tilde{\tau}}^{2} \tilde{D}^{\tau}=\operatorname{diag}\left(M_{\tilde{\tau}_{1}}^{2}, M_{\tilde{\tau}_{2}}^{2}, M_{\tilde{\tau}_{3}}^{2}, M_{\tilde{\tau}_{4}}^{2}, M_{\tilde{\tau}_{5}}^{2}, M_{\tilde{\tau}_{6}}^{2}, M_{\tilde{\tau}_{7}}^{2}, M_{\tilde{\tau}_{8}}^{2} M_{\tilde{\tau}_{9}}^{2}, M_{\tilde{\tau}_{10}}^{2}\right) .
$$

The mass-squared matrix in the sneutrino sector has a similar structure. In the basis

$$
\left(\tilde{\nu}_{\tau L}, \tilde{N}_{L}, \tilde{\nu}_{\tau R}, \tilde{N}_{R}, \tilde{\nu}_{\mu L}, \tilde{\nu}_{\mu R}, \tilde{\nu}_{e L}, \tilde{\nu}_{e R}, \tilde{\nu}_{4 L}, \tilde{\nu}_{4 R}\right),
$$

where the sneutrino mass squared matrix $\left(M_{\tilde{\nu}}^{2}\right)_{i j}=m_{i j}^{2}$ has elements given by

$$
\begin{aligned}
& m_{11}^{2}=\tilde{M}_{\tau L}^{2}+\frac{v_{2}^{2}}{2}\left|f_{1}^{\prime}\right|^{2}+\left|f_{3}\right|^{2}+\frac{1}{2} m_{Z}^{2} \cos 2 \beta, \quad m_{22}^{2}=\tilde{M}_{N}^{2}+\frac{v_{1}^{2}}{2}\left|f_{2}\right|^{2}+\left|f_{5}\right|^{2}+\left|f_{5}^{\prime}\right|^{2}+\left|f_{5}^{\prime \prime}\right|^{2}+\left|h_{8}\right|^{2}, \\
& m_{33}^{2}=\tilde{M}_{\nu_{\tau}}^{2}+\frac{v_{2}^{2}}{2}\left|f_{1}^{\prime}\right|^{2}+\left|f_{5}\right|^{2}, \quad m_{44}^{2}=\tilde{M}_{\chi}^{2}+\frac{v_{1}^{2}}{2}\left|f_{2}\right|^{2}+\left|f_{3}\right|^{2}+\left|f_{3}^{\prime}\right|^{2}+\left|f_{3}^{\prime \prime}\right|^{2}+\left|h_{6}\right|^{2}-\frac{1}{2} m_{Z}^{2} \cos 2 \beta, \\
& m_{55}^{2}=\tilde{M}_{\mu L}^{2}+\frac{v_{2}^{2}}{2}\left|h_{1}^{\prime}\right|^{2}+\left|f_{3}^{\prime}\right|^{2}+\frac{1}{2} m_{Z}^{2} \cos 2 \beta, \quad m_{66}^{2}=\tilde{M}_{\nu_{\mu}}^{2}+\frac{v_{2}^{2}}{2}\left|h_{1}^{\prime}\right|^{2}+\left|f_{5}^{\prime}\right|^{2} \\
& m_{77}^{2}=\tilde{M}_{e L}^{2}+\frac{v_{2}^{2}}{2}\left|h_{2}^{\prime}\right|^{2}+\left|f_{3}^{\prime \prime}\right|^{2}+\frac{1}{2} m_{Z}^{2} \cos 2 \beta, \quad m_{88}^{2}=\tilde{M}_{\nu_{e}}^{2}+\frac{v_{2}^{2}}{2}\left|h_{2}^{\prime}\right|^{2}+\left|f_{5}^{\prime \prime}\right|^{2}, \\
& m_{99}^{2}=\tilde{M}_{4 L}^{2}+\frac{v_{2}^{2}}{2}\left|y_{5}^{\prime}\right|^{2}+\left|h_{6}\right|^{2}+\frac{1}{2} m_{Z}^{2} \cos 2 \beta, \quad m_{1010}^{2}=\tilde{M}_{\nu 4}^{2}+\left|h_{8}\right|^{2}+\frac{v_{2}^{2}}{2}\left|y_{5}^{\prime}\right|^{2}, \quad m_{12}^{2}=m_{21}^{2 *}=\frac{v_{2} f_{5} f_{1}^{\prime *}}{\sqrt{2}}-\frac{v_{1} f_{2} f_{3}^{*}}{\sqrt{2}}, \\
& m_{13}^{2}=m_{31}^{2 *}=\frac{f_{1}^{\prime *}}{\sqrt{2}}\left(v_{2} A_{\nu_{\tau}}^{*}-\mu v_{1}\right), \quad m_{14}^{2}=m_{41}^{2 *}=0, \quad m_{15}^{2}=m_{51}^{2 *}=f_{3}^{\prime} f_{3}^{*}, \quad m_{16}^{2}=m_{61}^{2 *}=0, \\
& m_{17}^{2}=m_{71}^{2 *}=f_{3}^{\prime \prime} f_{3}^{*}, \quad m_{18}^{2}=m_{81}^{2 *}=0, \quad m_{23}^{2}=m_{32}^{2 *}=0, \quad m_{24}^{2}=m_{42}^{2 *}=\frac{f_{2}^{*}}{\sqrt{2}}\left(v_{1} A_{N}^{*}-\mu v_{2}\right), \\
& m_{25}^{2}=m_{52}^{2 *}=-\frac{v_{1} f_{2}^{*} f_{3}^{\prime}}{\sqrt{2}}+\frac{h_{1}^{\prime} v_{2} f_{5}^{\prime *}}{\sqrt{2}}, \quad m_{26}^{2}=m_{62}^{2 *}=0, \quad m_{27}^{2}=m_{72}^{2 *}=-\frac{v_{1} f_{2}^{*} f_{3}^{\prime \prime}}{\sqrt{2}}+\frac{h_{2}^{\prime} v_{2} f_{5}^{\prime \prime *}}{\sqrt{2}}, \\
& m_{28}^{2}=m_{82}^{2 *}=0, \quad m_{34}^{2}=m_{43}^{2 *}=\frac{v_{1} f_{2}^{*} f_{5}}{\sqrt{2}}-\frac{v_{2} f_{1}^{\prime} f_{3}^{*}}{\sqrt{2}}, \quad m_{35}^{2}=m_{53}^{2 *}=0, \quad m_{36}^{2}=m_{63}^{2 *}=f_{5} f_{5}^{\prime *}, \\
& m_{37}^{2}=m_{73}^{2 *}=0, \quad m_{38}^{2}=m_{83}^{2 *}=f_{5} f_{5}^{\prime \prime *}, \quad m_{45}^{2}=m_{54}^{2 *}=0, \\
& m_{46}^{2}=m_{64}^{2 *}=-\frac{h_{1}^{\prime *} v_{2} f_{3}^{\prime}}{\sqrt{2}}+\frac{v_{1} f_{2} f_{5}^{\prime *}}{\sqrt{2}}, \quad m_{47}^{2}=m_{74}^{2 *}=0, \\
& m_{48}^{2}=m_{84}^{2 *}=\frac{v_{1} f_{2} f_{5}^{\prime \prime *}}{\sqrt{2}}-\frac{v_{2} h_{2}^{\prime *} f_{3}^{\prime \prime}}{\sqrt{2}}, \quad m_{56}^{2}=m_{65}^{2 *}=\frac{h_{1}^{\prime *}}{\sqrt{2}}\left(v_{2} A_{\nu_{\mu}}^{*}-\mu v_{1}\right), \\
& m_{57}^{2}=m_{75}^{2 *}=f_{3}^{\prime \prime} f_{3}^{\prime *}, \quad m_{58}^{2}=m_{85}^{2 *}=0, \quad m_{67}^{2}=m_{76}^{2 *}=0, \quad m_{68}^{2}=m_{86}^{2 *}=f_{5}^{\prime} f_{5}^{\prime \prime *}, \quad m_{78}^{2}=m_{87}^{2 *}=\frac{h_{2}^{\prime *}}{\sqrt{2}}\left(v_{2} A_{\nu_{e}}^{*}-\mu v_{1}\right), \\
& m_{19}^{2}=m_{91}^{2 *}=h_{6} f_{3}^{*}, \quad m_{110}^{2}=m_{101}^{2 *}=0, \quad m_{29}^{2}=m_{92}^{2 *}=-\frac{f_{2} v_{1} h_{6}}{\sqrt{2}}+\frac{v_{2} h_{8} y_{5}^{*}}{\sqrt{2}}, \quad m_{210}^{2}=m_{102}^{2 *}=0, \\
& m_{39}^{2}=m_{93}^{2 *}=0, \quad m_{310}^{2}=m_{103}^{2 *}=f_{5} h_{8}^{*}, \quad m_{49}^{2}=m_{94}^{2 *}=0, \quad m_{410}^{2}=m_{104}^{2 *}=-\frac{v_{2} y_{5}^{\prime} h_{6}}{\sqrt{2}}+\frac{v_{1} h_{8}^{*} f_{2}}{\sqrt{2}}, \\
& m_{59}^{2}=m_{95}^{2 *}=h_{6} f_{3}^{* *}, \quad m_{510}^{2}=m_{105}^{2 *}=0, \quad m_{69}^{2}=m_{96}^{2 *}=0, \quad m_{610}^{2}=m_{106}^{2 *}=f_{5}^{\prime} h_{8}^{*}, \\
& m_{79}^{2}=m_{97}^{2 *}=h_{6} f_{3}^{\prime \prime *}, \quad m_{710}^{2}=m_{107}^{2 *}=0, \quad m_{89}^{2}=m_{98}^{2 *}=0, \quad m_{810}^{2}=m_{108}^{2 *}=f_{5}^{\prime \prime} h_{8}^{*}, \\
& m_{910}^{2}=m_{109}^{2 *}=\frac{y_{5}^{\prime}}{\sqrt{2}}\left(v_{2} A_{4 \nu}^{*}-\mu v_{1}\right) \text {. }
\end{aligned}
$$

Again as in the charged slepton sector we assume that all the masses are of the electroweak size so all the terms enter in the mass-squared matrix. This mass-squared matrix can be diagonalized by the unitary transformation,

$$
\tilde{D}^{\nu \dagger} M_{\tilde{\nu}}^{2} \tilde{D}^{\nu}=\operatorname{diag}\left(M_{\tilde{\nu}_{1}}^{2}, M_{\tilde{\nu}_{2}}^{2}, M_{\tilde{\nu}_{3}}^{2}, M_{\tilde{\nu}_{4}}^{2}, M_{\tilde{\nu}_{5}}^{2}, M_{\tilde{\nu}_{6}}^{2}, M_{\tilde{\nu}_{7}}^{2}, M_{\tilde{\nu}_{8}}^{2}, M_{\tilde{\nu}_{9}}^{2}, M_{\tilde{\nu}_{10}}^{2}\right) .
$$




\section{APPENDIX B: INTERACTIONS THAT ENTER IN THE ANALYSES OF THE RADIATIVE DECAYS, OF THE EDMS AND OF THE MAGNETIC DIPOLE MOMENTS OF THE LEPTONS}

In this Appendix we discuss the interactions in the mass diagonal basis involving charged leptons, sneutrinos and charginos. Thus we have

$-\mathcal{L}_{\tau \tilde{\nu} \tilde{\chi}^{-}}=\sum_{i=1}^{2} \sum_{j=1}^{10} \bar{\tau}_{\alpha}\left(C_{\alpha i j}^{L} P_{L}+C_{\alpha i j}^{R} P_{R}\right) \tilde{\chi}^{c i} \tilde{\nu}_{j}+$ H.c.,

such that

$$
\begin{aligned}
C_{\alpha i j}^{L}= & g\left(-\kappa_{\tau} U_{i 2}^{*} D_{R 1 \alpha}^{\tau *} \tilde{D}_{1 j}^{\nu}-\kappa_{\mu} U_{i 2}^{*} D_{R 3 \alpha}^{\tau *} \tilde{D}_{5 j}^{\nu}-\kappa_{e} U_{i 2}^{*} D_{R 4 \alpha}^{\tau *} \tilde{D}_{7 j}^{\nu}\right. \\
& \left.-\kappa_{4 \ell} U_{i 2}^{*} D_{R 5 \alpha}^{\tau *} \tilde{D}_{9 j}^{\nu}+U_{i 1}^{*} D_{R 2 \alpha}^{\tau *} \tilde{D}_{4 j}^{\nu}-\kappa_{N} U_{i 2}^{*} D_{R 2 \alpha}^{\tau *} \tilde{D}_{2 j}^{\nu}\right)
\end{aligned}
$$

$$
\begin{aligned}
C_{\alpha i j}^{R}= & g\left(-\kappa_{\nu_{\tau}} V_{i 2} D_{L 1 \alpha}^{\tau *} \tilde{D}_{3 j}^{\nu}-\kappa_{\nu_{\mu}} V_{i 2} D_{L 3 \alpha}^{\tau *} \tilde{D}_{6 j}^{\nu}\right. \\
& -\kappa_{\nu_{e}} V_{i 2} D_{L 4 \alpha}^{\tau *} \tilde{D}_{8 j}^{\nu}+V_{i 1} D_{L 1 \alpha}^{\tau *} \tilde{D}_{1 j}^{\nu}+V_{i 1} D_{L 3 \alpha}^{\tau *} \tilde{D}_{5 j}^{\nu} \\
& \left.-\kappa_{\nu_{4}} V_{i 2} D_{L 5 \alpha}^{\tau *} \tilde{D}_{10 j}^{\nu}+V_{i 1} D_{L 4 \alpha}^{\tau *} \tilde{D}_{7 j}^{\nu}-\kappa_{E} V_{i 2} D_{L 2 \alpha}^{\tau *} \tilde{D}_{4 j}^{\nu}\right),
\end{aligned}
$$

where $D_{L, R}^{\tau}$ and $\tilde{D}^{\nu}$ are the charged lepton and sneutrino diagonalizing matrices and are defined by Eqs. (13) and (A7), respectively, and $U$ and $V$ are the matrices that diagonalize the chargino mass matrix $M_{C}$ so that [56]

$$
U^{*} M_{C} V^{-1}=\operatorname{diag}\left(m_{\chi_{1}^{ \pm}} m_{\chi_{2}^{ \pm}}\right) .
$$

Further,

$$
\begin{gathered}
\left(\kappa_{N}, \kappa_{\tau}, \kappa_{\mu}, \kappa_{e}, \kappa_{4 \ell}\right)=\frac{\left(m_{N}, m_{\tau}, m_{\mu}, m_{e}, m_{4 \ell}\right)}{\sqrt{2} m_{W} \cos \beta}, \\
\left(\kappa_{E}, \kappa_{\nu_{\tau}}, \kappa_{\nu_{\mu}}, \kappa_{\nu_{e}}, \kappa_{\nu_{4}}\right)=\frac{\left(m_{E}, m_{\nu_{\tau}}, m_{\nu_{\mu}}, m_{\nu_{e}}, m_{\nu_{4}}\right)}{\sqrt{2} m_{W} \sin \beta} .
\end{gathered}
$$

where $m_{W}$ is the mass of the $W$ boson and $\tan \beta=$ $\left\langle H_{2}^{2}\right\rangle /\left\langle H_{1}^{1}\right\rangle$ where $H_{1}, H_{2}$ are the two Higgs doublets of MSSM.

We now discuss the interactions in the mass diagonal basis involving charged leptons, sleptons and neutralinos. Thus we have

$-\mathcal{L}_{\tau \tilde{\tau} \tilde{\chi}^{0}}=\sum_{i=1}^{4} \sum_{j=1}^{10} \bar{\tau}_{\alpha}\left(C_{\alpha i j}^{\prime L} P_{L}+C_{\alpha i j}^{\prime R} P_{R}\right) \tilde{\chi}_{i}^{0} \tilde{\tau}_{j}+$ H.c.,

such that

$$
\begin{aligned}
C_{\alpha i j}^{\prime L}= & \sqrt{2}\left(\alpha_{\tau i} D_{R 1 \alpha}^{\tau *} \tilde{D}_{1 j}^{\tau}-\delta_{E i} D_{R 2 \alpha}^{\tau *} \tilde{D}_{2 j}^{\tau}-\gamma_{\tau i} D_{R 1 \alpha}^{\tau *} \tilde{D}_{3 j}^{\tau}\right. \\
& +\beta_{E i} D_{R 2 \alpha}^{\tau *} \tilde{D}_{4 j}^{\tau}+\alpha_{\mu i} D_{R 3 \alpha}^{\tau *} \tilde{D}_{5 j}^{\tau}-\gamma_{\mu i} D_{R 3 \alpha}^{\tau *} \tilde{D}_{6 j}^{\tau} \\
& +\alpha_{e i} D_{R 4 \alpha}^{\tau *} \tilde{D}_{7 j}^{\tau}-\gamma_{e i} D_{R 4 \alpha}^{\tau *} \tilde{D}_{8 j}^{\tau}+\alpha_{4 \ell i} D_{R 5 \alpha}^{\tau *} \tilde{D}_{9 j}^{\tau} \\
& \left.-\gamma_{4 \ell i} D_{R 5 \alpha}^{\tau *} \tilde{D}_{10 j}^{\tau}\right), \\
C_{\alpha i j}^{\prime R}= & \sqrt{2}\left(\beta_{\tau i} D_{L 1 \alpha}^{\tau *} \tilde{D}_{1 j}^{\tau}-\gamma_{E i} D_{L 2 \alpha}^{\tau *} \tilde{D}_{2 j}^{\tau}-\delta_{\tau i} D_{L 1 \alpha}^{\tau *} \tilde{D}_{3 j}^{\tau}\right. \\
& +\alpha_{E i} D_{L 2 \alpha}^{\tau *} \tilde{D}_{4 j}^{\tau}+\beta_{\mu i} D_{L 3 \alpha}^{\tau *} \tilde{D}_{5 j}^{\tau}-\delta_{\mu i} D_{L 3 \alpha}^{\tau *} \tilde{D}_{6 j}^{\tau} \\
& +\beta_{e i} D_{L 4 \alpha}^{\tau *} \tilde{D}_{7 j}^{\tau}-\delta_{e i} D_{L 4 \alpha}^{\tau *} \tilde{D}_{8 j}^{\tau} \\
& \left.+\beta_{4 \ell i} D_{L 5 \alpha}^{\tau *} \tilde{D}_{9 j}^{\tau}-\delta_{4 \ell i} D_{L 5 \alpha}^{\tau *} \tilde{D}_{10 j}^{\tau}\right),
\end{aligned}
$$

where

$\alpha_{E i}=\frac{g m_{E} X_{4 i}^{*}}{2 m_{W} \sin \beta} ; \quad \beta_{E i}=e X_{1 i}^{\prime}+\frac{g}{\cos \theta_{W}} X_{2 i}^{\prime}\left(\frac{1}{2}-\sin ^{2} \theta_{W}\right)$

$\gamma_{E i}=e X_{1 i}^{* *}-\frac{g \sin ^{2} \theta_{W}}{\cos \theta_{W}} X_{2 i}^{\prime *} ; \quad \delta_{E i}=-\frac{g m_{E} X_{4 i}}{2 m_{W} \sin \beta}$

and

$\alpha_{\tau i}=\frac{g m_{\tau} X_{3 i}}{2 m_{W} \cos \beta} ; \quad \alpha_{\mu i}=\frac{g m_{\mu} X_{3 i}}{2 m_{W} \cos \beta} ;$

$\alpha_{e i}=\frac{g m_{e} X_{3 i}}{2 m_{W} \cos \beta} ; \quad \alpha_{4 \ell i}=\frac{g m_{4 \ell} X_{3 i}}{2 m_{W} \cos \beta}$,

$\delta_{\tau i}=-\frac{g m_{\tau} X_{3 i}^{*}}{2 m_{W} \cos \beta} ; \quad \delta_{\mu i}=-\frac{g m_{\mu} X_{3 i}^{*}}{2 m_{W} \cos \beta} ;$

$\delta_{e i}=-\frac{g m_{e} X_{3 i}^{*}}{2 m_{W} \cos \beta} ; \quad \delta_{4 \ell i}=-\frac{g m_{4 \ell} X_{3 i}^{*}}{2 m_{W} \cos \beta}$,

and where

$$
\begin{gathered}
\beta_{\tau i}=\beta_{\mu i}=\beta_{e i}=\beta_{4 \ell i}=-e X_{1 i}^{\prime *}+\frac{g}{\cos \theta_{W}} X_{2 i}^{\prime *}\left(-\frac{1}{2}+\sin ^{2} \theta_{W}\right), \\
\gamma_{\tau i}=\gamma_{\mu i}=\gamma_{e i}=\gamma_{4 \ell i}=-e X_{1 i}^{\prime}+\frac{g \sin ^{2} \theta_{W}}{\cos \theta_{W}} X_{2 i}^{\prime} .
\end{gathered}
$$

Here $X^{\prime}$ are defined by

$$
\begin{gathered}
X_{1 i}^{\prime}=X_{1 i} \cos \theta_{W}+X_{2 i} \sin \theta_{W}, \\
X_{2 i}^{\prime}=-X_{1 i} \sin \theta_{W}+X_{2 i} \cos \theta_{W},
\end{gathered}
$$

where $X$ diagonalizes the neutralino mass matrix, i.e.,

$$
X^{T} M_{\chi^{0}} X=\operatorname{diag}\left(m_{\chi_{1}^{0}}, m_{\chi_{2}^{0}}, m_{\chi_{3}^{0}}, m_{\chi_{4}^{0}}\right) .
$$


Further, $\tilde{D}^{\tau}$ that enter in Eqs. (B8) and (B9) is a matrix which diagonalizes the charged slepton mass squared matrix and is defined in Eq. (A4).

In addition to the supersymmetric loop diagrams, we compute the contributions arising from the exchange of $W$ and $Z$ bosons and leptons and mirror leptons in the loops. For the $W$ boson exchange the interactions are given by

$-\mathcal{L}_{\tau W \psi}=W_{\rho}^{\dagger} \sum_{i=1}^{5} \sum_{\alpha=1}^{5} \bar{\psi}_{i} \gamma^{\rho}\left(C_{L i \alpha}^{W} P_{L}+C_{R i \alpha}^{W} P_{R}\right) \tau_{\alpha}+$ H.c.

where

$C_{L_{i \alpha}}^{W}=\frac{g}{\sqrt{2}}\left[D_{L 1 i}^{\nu *} D_{L 1 \alpha}^{\tau}+D_{L 3 i}^{\nu *} D_{L 3 \alpha}^{\tau}+D_{L 4 i}^{\nu *} D_{L 4 \alpha}^{\tau}+D_{L 5 i}^{\nu *} D_{L 5 \alpha}^{\tau}\right]$,

and

$$
C_{R_{i \alpha}}^{W}=\frac{g}{\sqrt{2}}\left[D_{R 2 i}^{\nu *} D_{R 2 \alpha}^{\tau}\right]
$$

Here $D_{L, R}^{\nu}$ are matrices of a biunitary transformation that diagonalizes the neutrino mass matrix and are defined in
Eq. (9). For the $Z$ boson exchange the interactions that enter are given by

$-\mathcal{L}_{\tau \tau Z}=Z_{\rho} \sum_{\alpha=1}^{5} \sum_{\beta=1}^{5} \bar{\tau}_{\alpha} \gamma^{\rho}\left(C_{L \alpha \beta}^{Z} P_{L}+C_{R \alpha \beta}^{Z} P_{R}\right) \tau_{\beta}$,

where

$$
\begin{aligned}
C_{L_{\alpha \beta}}^{Z}= & \frac{g}{\cos \theta_{W}}\left[x \left(D_{L \alpha 1}^{\tau \dagger} D_{L 1 \beta}^{\tau}+D_{L \alpha 2}^{\tau \dagger} D_{L 2 \beta}^{\tau}+D_{L \alpha 3}^{\tau \dagger} D_{L 3 \beta}^{\tau}\right.\right. \\
& \left.+D_{L \alpha 4}^{\tau \dagger} D_{L 4 \beta}^{\tau}+D_{L \alpha 5}^{\tau \dagger} D_{L 5 \beta}^{\tau}\right) \\
& -\frac{1}{2}\left(D_{L \alpha 1}^{\tau \dagger} D_{L 1 \beta}^{\tau}+D_{L \alpha 3}^{\tau \dagger} D_{L 3 \beta}^{\tau}+D_{L \alpha 4}^{\tau \dagger} D_{L 4 \beta}^{\tau}\right. \\
& \left.\left.+D_{L \alpha 5}^{\tau \dagger} D_{L 5 \beta}^{\tau}\right)\right],
\end{aligned}
$$

and

$$
\begin{aligned}
C_{R_{\alpha \beta}}^{Z}= & \frac{g}{\cos \theta_{W}}\left[x \left(D_{R \alpha 1}^{\tau \dagger} D_{R 1 \beta}^{\tau}+D_{R \alpha 2}^{\tau^{\dagger}} D_{R 2 \beta}^{\tau}+D_{R \alpha 3}^{\tau \dagger} D_{R 3 \beta}^{\tau}\right.\right. \\
& \left.\left.+D_{R \alpha 4}^{\tau \dagger} D_{R 4 \beta}^{\tau}+D_{R \alpha 5}^{\tau \dagger} D_{R 5 \beta}^{\tau}\right)-\frac{1}{2}\left(D_{R \alpha 2}^{\tau \dagger} D_{R 2 \beta}^{\tau}\right)\right] .
\end{aligned}
$$

with $x=\sin ^{2} \theta_{W}$.
[1] J. Adam et al. (MEG Collaboration), New Constraint on the Existence of the $\mu^{+} \rightarrow e^{+} \gamma$ Decay, Phys. Rev. Lett. 110, 201801 (2013).

[2] B. Aubert et al. (BABAR Collaboration), Searches for Lepton Flavor Violation in the Decays $\tau+-\rightarrow e+-\gamma$ and $\tau+-\rightarrow \mu+-\gamma$, Phys. Rev. Lett. 104, 021802 (2010).

[3] K. Hayasaka et al. (Belle Collaboration), New search for $\tau \rightarrow$ $\mu \gamma$ and $\tau \rightarrow e \gamma$ decays at Belle, Phys. Lett. B 666, 16 (2008).

[4] J. Baron et al. (ACME Collaboration), Methods, analysis, and the treatment of systematic errors for the electron electric dipole moment search in thorium monoxide, New J. Phys. 19, 073029 (2017).

[5] J. Beringer et al. (Particle Data Group), Review of particle physics (RPP), Phys. Rev. D 86, 010001 (2012).

[6] G. W. Bennett et al. (Muon $(g-2)$ Collaboration), An improved limit on the muon electric dipole moment, Phys. Rev. D 80, 052008 (2009).

[7] H. Georgi, Towards a grand unified theory of flavor, Nucl. Phys. B156, 126 (1979); F. Wilczek and A. Zee, Families from spinors, Phys. Rev. D 25, 553 (1982); G. Senjanovic, F. Wilczek, and A. Zee, Reflections on mirror fermions, Phys. Lett. B 141, 389 (1984); J. Maalampi, J. T. Peltoniemi, and M. Roos, The magnetic moment of the electron neutrino in mirror models, Phys. Lett. B 220, 441 (1989); B. Kors and P. Nath, Effective action and soft supersymmetry breaking for intersecting D-brane models, Nucl. Phys. B681, 77 (2004); K. S. Babu, I. Gogoladze, P. Nath, and R. M. Syed, Fermion mass generation in $\mathrm{SO}(10)$ with a unified Higgs sector, Phys. Rev. D 74, 075004 (2006); A Unied framework for symmetry breaking in SO(10), Phys. Rev. D72, 095011 (2006).

[8] T. P. T. Dijkstra, L. R. Huiszoon, and A. N. Schellekens, Supersymmetric standard model spectra from RCFT orientifolds, Nucl. Phys. B710, 3 (2005).

[9] O. Lebedev, H. P. Nilles, S. Raby, S. Ramos-Sanchez, M. Ratz, P. K. S. Vaudrevange, and A. Wingerter, A minilandscape of exact MSSM spectra in heterotic orbifolds, Phys. Lett. B 645, 88 (2007).

[10] T. Ibrahim and P. Nath, Large tau and tau neutrino electric dipole moments in models with vectorlike multiplets, Phys. Rev. D 81, 033007 (2010); Erratum, Phys. Rev. D 89, 119902(E) (2014).

[11] T. Ibrahim and P. Nath, $\tau \rightarrow \mu \gamma$ decay in extensions with a vectorlike generation, Phys. Rev. D 87, 015030 (2013).

[12] A. Aboubrahim, T. Ibrahim, and P. Nath, Radiative decays of cosmic background neutrinos in extensions of MSSM with a vectorlike lepton generation, Phys. Rev. D 88, 013019 (2013).

[13] T. Ibrahim, A. Itani, and P. Nath, $\mu \rightarrow e \gamma$ decay in an MSSM extension, Phys. Rev. D 92, 015003 (2015). 
[14] S. P. Martin, Extra vectorlike matter and the lightest Higgs scalar boson mass in low-energy supersymmetry, Phys. Rev. D 81, 035004 (2010).

[15] P. W. Graham, A. Ismail, S. Rajendran, and P. Saraswat, A little solution to the little hierarchy problem: A vectorlike generation, Phys. Rev. D 81, 055016 (2010).

[16] S. P. Martin, Raising the Higgs mass with Yukawa couplings for isotriplets in vectorlike extensions of minimal supersymmetry, Phys. Rev. D 82, 055019 (2010).

[17] S. P. Martin and J. D. Wells, Implications of gauge-mediated supersymmetry breaking with vectorlike quarks and a 125 GeV Higgs boson, Phys. Rev. D 86, 035017 (2012).

[18] T. Moroi, R. Sato, and T. T. Yanagida, Extra matters decree the relatively heavy Higgs of mass about $125 \mathrm{GeV}$ in the supersymmetric model, Phys. Lett. B 709, 218 (2012).

[19] W. Fischler and W. Tangarife, Vectorlike fields, messenger mixing and the Higgs mass in gauge mediation, J. High Energy Phys. 05 (2014) 151.

[20] M. Endo, K. Hamaguchi, S. Iwamoto, and N. Yokozaki, Higgs mass, muon $g-2$, and LHC prospects in gauge mediation models with vectorlike matters, Phys. Rev. D 85, 095012 (2012).

[21] F. del Aguila, J. de Blas, and M. Perez-Victoria, Effects of new leptons in electroweak precision data, Phys. Rev. D 78, 013010 (2008).

[22] N. Arkani-Hamed, K. Blum, R. T. D'Agnolo, and J. Fan, 2:1 for naturalness at the LHC?, J. High Energy Phys. 01 (2013) 149.

[23] J. Kearney, A. Pierce, and N. Weiner, Vectorlike fermions and Higgs couplings, Phys. Rev. D 86, 113005 (2012).

[24] A. Joglekar, P. Schwaller, and C. E. M. Wagner, Dark matter and enhanced Higgs to diphoton rate from vectorlike leptons, J. High Energy Phys. 12 (2012) 064.

[25] K. Ishiwata and M. B. Wise, Phenomenology of heavy vectorlike leptons, Phys. Rev. D 88, 055009 (2013).

[26] K. Ishiwata, Z. Ligeti, and M. B. Wise, New vectorlike fermions and flavor physics, J. High Energy Phys. 10 (2015) 027.

[27] S. A. R. Ellis, R. M. Godbole, S. Gopalakrishna, and J. D. Wells, Survey of vectorlike fermion extensions of the Standard Model and their phenomenological implications, J. High Energy Phys. 09 (2014) 130.

[28] B. O'Leary et al. (SuperB Collaboration), SuperB progress reports-Physics, arXiv:1008.1541.

[29] T. Aushev et al., Physics at Super B Factory, arXiv: 1002.5012 .

[30] M.E. Biagini et al. (SuperB Collaboration), SuperB progress reports: The collider, arXiv:1009.6178.

[31] J. L. Hewett et al., Fundamental physics at the intensity frontier, arXiv:1205.2671.

[32] F. Gabbiani and A. Masiero, FCNC in generalized supersymmetric theories, Nucl. Phys. B322, 235 (1989).

[33] R. L. Arnowitt and P. Nath, $\mu \rightarrow e \gamma$ and $\tau \rightarrow \mu \gamma$ Decays in String Models with E(6) Symmetry, Phys. Rev. Lett. 66, 2708 (1991).

[34] F. Gabbiani, E. Gabrielli, A. Masiero, and L. Silvestrini, A complete analysis of FCNC and $C P$ constraints in general SUSY extensions of the standard model, Nucl. Phys. B477, 321 (1996).
[35] A. Abada, C. Biggio, F. Bonnet, M. B. Gavela, and T. Hambye, $\mu \rightarrow e \gamma$ and $\tau \rightarrow l \gamma$ decays in the fermion triplet seesaw model, Phys. Rev. D 78, 033007 (2008).

[36] W. Altmannshofer, A. J. Buras, S. Gori, P. Paradisi, and D. M. Straub, Anatomy and phenomenology of FCNC and CPV effects in SUSY theories, Nucl. Phys. B830, 17 (2010).

[37] D. McKeen, M. Pospelov, and A. Ritz, Electric dipole moment signatures of PeV-scale superpartners, Phys. Rev. D 87, 113002 (2013).

[38] R. Escribano and E. Masso, Improved bounds on the electromagnetic dipole moments of the $\tau$ lepton, Phys. Lett. B 395, 369 (1997).

[39] C. Patrignani et al. (Particle Data Group), Review of particle physics, Chin. Phys. C 40, 100001 (2016).

[40] M. Lindner, M. Platscher, and F. S. Queiroz, A call for new physics: The muon anomalous magnetic moment and lepton flavor violation, Phys. Rep. 731, 1 (2018).

[41] A. Crivellin, M. Hoferichter, and P. Schmidt-Wellenburg, Combined explanations of $(g-2)_{\mu, e}$ and implications for a large muon EDM, arXiv:1807.11484.

[42] M. Aaboud et al. (ATLAS Collaboration), Measurements of Higgs boson properties in the diphoton decay channel with $36 \mathrm{fb}^{-1}$ of $p p$ collision data at $\sqrt{s}=13 \mathrm{TeV}$ with the ATLAS detector, Phys. Rev. D 98, 052005 (2018).

[43] A. M. Sirunyan et al. (CMS Collaboration), Measurements of Higgs boson properties in the diphoton decay channel in proton-proton collisions at $\sqrt{s}=13 \mathrm{TeV}$, arXiv: 1804.02716.

[44] W. Z. Feng and P. Nath, Higgs diphoton rate and mass enhancement with vectorlike leptons and the scale of supersymmetry, Phys. Rev. D 87, 075018 (2013).

[45] R. Dermisek and A. Raval, Explanation of the muon $g-2$ anomaly with vectorlike leptons and its implications for Higgs decays, Phys. Rev. D 88, 013017 (2013); Z. Poh and S. Raby, Vectorlike leptons: Muon $g-2$ anomaly, lepton flavor violation, Higgs boson decays, and lepton nonuniversality, Phys. Rev. D 96, 015032 (2017); A. Choudhury, L. Darm, L. Roszkowski, E. M. Sessolo, and S. Trojanowski, Muon $g-2$ and related phenomenology in constrained vectorlike extensions of the MSSM, J. High Energy Phys. 05 (2017) 072.

[46] A. Aboubrahim, T. Ibrahim, and P. Nath, Leptonic $g-2$ moments, $C P$ phases and the Higgs boson mass constraint, Phys. Rev. D 94, 015032 (2016).

[47] P. Nath, $C P$ Violation via Electroweak Gauginos and the Electric Dipole Moment of the Electron, Phys. Rev. Lett. 66, 2565 (1991).

[48] Y. Kizukuri and N. Oshimo, The neutron and electron electric dipole moments in supersymmetric theories, Phys. Rev. D 46, 3025 (1992).

[49] T. Ibrahim and P. Nath, The neutron and the lepton EDMs in MSSM, large $C P$ violating phases, and the cancellation mechanism, Phys. Rev. D 58, 111301 (1998); Erratum, Phys. Rev. D 60, 099902(E) (1999).

[50] T. Ibrahim and P. Nath, The neutron and the electron electric dipole moment in $N=1$ supergravity unification, Phys. Rev. D 57, 478 (1998); Erratum, Phys. Rev. D 58, 019901(E) (1998); Erratum, Phys. Rev. D 60, 079903(E) (1999); Erratum, Phys. Rev. D 60, 119901(E) (1999). 
[51] T. Falk and K. A. Olive, More on electric dipole moment constraints on phases in the constrained MSSM, Phys. Lett. B 439, 71 (1998).

[52] M. Brhlik, G. J. Good, and G. L. Kane, Electric dipole moments do not require the $C P$ violating phases of supersymmetry to be small, Phys. Rev. D 59, 115004 (1999).

[53] T. Ibrahim and P. Nath, Large $C P$ phases and the cancellation mechanism in EDMs in SUSY, string and brane models, Phys. Rev. D 61, 093004 (2000).

[54] T. Ibrahim and P. Nath, The chromoelectric dipole moment of the top quark in models with vectorlike multiplets, Phys. Rev. D 84, 015003 (2011).

[55] M. E. Gomez, T. Ibrahim, P. Nath, and S. Skadhauge, An improved analysis of $b \rightarrow s \gamma$ in supersymmetry, Phys. Rev. D 74, 015015 (2006).

[56] T. Ibrahim and P. Nath, $C P$ violation from Standard Model to strings, Rev. Mod. Phys. 80, 577 (2008).

[57] A. H. Chamseddine, R. L. Arnowitt, and P. Nath, Locally Supersymmetric Grand Unification, Phys. Rev. Lett. 49, 970 (1982).

[58] B. C. Allanach, SOFTSUSY: A program for calculating supersymmetric spectra, Comput. Phys. Commun. 143, 305 (2002).
[59] B. C. Allanach, S. P. Martin, D. G. Robertson, and R. Ruiz de Austri, The inclusion of two-loop SUSYQCD corrections to gluino and squark pole masses in the minimal and next-to-minimal supersymmetric Standard Model: SOFTSUSY3.7, Comput. Phys. Commun. 219, 339 (2017).

[60] W. Altmannshofer, R. Harnik, and J. Zupan, Low energy probes of PeV scale sfermions, J. High Energy Phys. 11 (2013) 202.

[61] U. Bellgardt et al. (SINDRUM Collaboration), Search for the decay $\mu+\rightarrow e+e+e-$, Nucl. Phys. B299, 1 (1988).

[62] A. M. Baldini et al., MEG upgrade proposal, arXiv: 1301.7225.

[63] S. A. R. Ellis and A. Pierce, Impact of future lepton flavor violation measurements in the minimal supersymmetric Standard Model, Phys. Rev. D 94, 015014 (2016).

[64] W. H. Bertl et al. (SINDRUM II Collaboration), A search for muon to electron conversion in muonic gold, Eur. Phys. J. C 47, 337 (2006).

[65] R. J. Abrams et al. (Mu2e Collaboration), Mu2e conceptual design report, arXiv:1211.7019. 